\title{
GAWPS: A MRST-based module for wellbore profiling and graphical analysis of flow units
}

\author{
Gustavo P. Oliveira ${ }^{1 \oplus *}$, Thiago N. E. Rodrigues ${ }^{1}$, Knut-Andreas Lie ${ }^{2 \oplus}$ \\ ${ }^{1}$ TRIL Lab, Graduate Program in Mechanical Engineering, Federal University of Paraíba, João Pessoa, Brazil \\ ${ }^{2}$ Mathematics and Cybernetics, SINTEF Digital, Oslo, Norway
}

\section{Keywords:}

Reservoir modeling

hydraulic flow unit

graphical methods

data analysis

Cited as:

Oliveira, G. P., Rodrigues, T. N. E., Lie, K.-A. GAWPS: A MRST-based module for wellbore profiling and graphical analysis of flow units. Advances in Geo-Energy Research, 2021, 6(1): 38-53. https://doi.org/10.46690/ager.2022.01.04

\begin{abstract}
:
Several graphical methods have been developed to understand the stratigraphy observed in wells and assist experts in estimating rock quality, defining limits for barriers, baffles, and speed zones, and in particular, delineating hydraulic flow units. At present, there exists no computational tool that bundles the main graphical methods used for defining flow units. This paper introduces an add-on module to the MATLAB Reservoir Simulation Toolbox that contains computational routines to carry out such graphical analyses, both qualitatively and quantitatively. We also describe a new secondary method defined as the derivative of the stratigraphic modified Lorenz plot, which we use to classify depth ranges within the reservoir into barriers, strong baffles, weak baffles, and normal units, based on flow unit speed over those depths. We demonstrate the capabilities of the "Graphical Analysis for Well Placement Strategy" module by applying it to several case studies of both real and synthetic reservoirs.
\end{abstract}

\section{Introduction}

Petrophysics can translate raw data into descriptions of lithology, volumetrics, and other attributes by bringing together knowledge regarding rock and fluid interactions in oil and gas reservoirs. This information is incorporated into an overall reservoir characterization and can subsequently be used to build and deploy simulation models (Cannon, 2015).

One important part of reservoir characterization is to delineate flow units. Several graphical tools have been developed to aid this process, namely the Winland plot, the stratigraphic flow profile, the stratigraphic modified Lorenz plot, and the modified Lorenz plot (Gunter et al., 1997a). These four tools were embedded into the so-called petrophysical integration process model (PIPM) as a subprocess aimed at detecting compartments and flow units (Gunter et al., 1997b).

Later, PIPM was adapted for specific applications in unconventional natural gas reservoirs, but the graphical tools remained unchanged (Newsham and Rushing, 2001; Rushing and Newsham, 2001). The multi-component stratigraphic modified
Lorenz plot (MCSMLP) was devised to aggregate petrophysical rock types, bulk volume of water and hydrocarbon, as well as total storage capacity and flow capacity, by searching for a quick evaluation of undeveloped and untested flow zones (Gunter et al., 2012). Recently, the role played by graphical methods in flow unit delineation has become more evident with the development of a well-structured workflow focused on petrophysical rock type verification (Gunter et al., 2017, 2018). As far as we know, there are no open-source modules or packages that offer routines for computing these quantitative profiles for quick statistical verification.

This paper approaches the issue from two directions. Firstly, we introduce the GAWPS module (the name is an abbreviation for "Graphical Analysis for Well Placement Strategy"), which can be used to compute a class of quantitative profiles across pre-selected wells for flow unit analysis. The module is built on top of the MATLAB Reservoir Simulation Toolbox (MRST) (Lie, 2019), an open-source toolbox for reservoir simulation (available on availableonhttp://mrst.no) based on

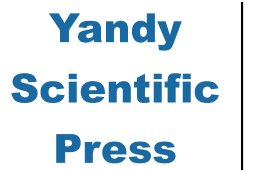

${ }^{*}$ Corresponding author.

E-mail address: gustavo.oliveira@ci.ufpb.br (G. P. Oliveira); thiago.ney@academico.ufpb.br (T. N. E. Rodrigues);

Knut-Andreas.Lie@sintef.no (K.-A. Lie).

2207-9963 (C) The Author(s) 2021.

Received November 19, 2021; revised December 14, 2021; accepted December 15, 2021; available online December 20 , 2021. 


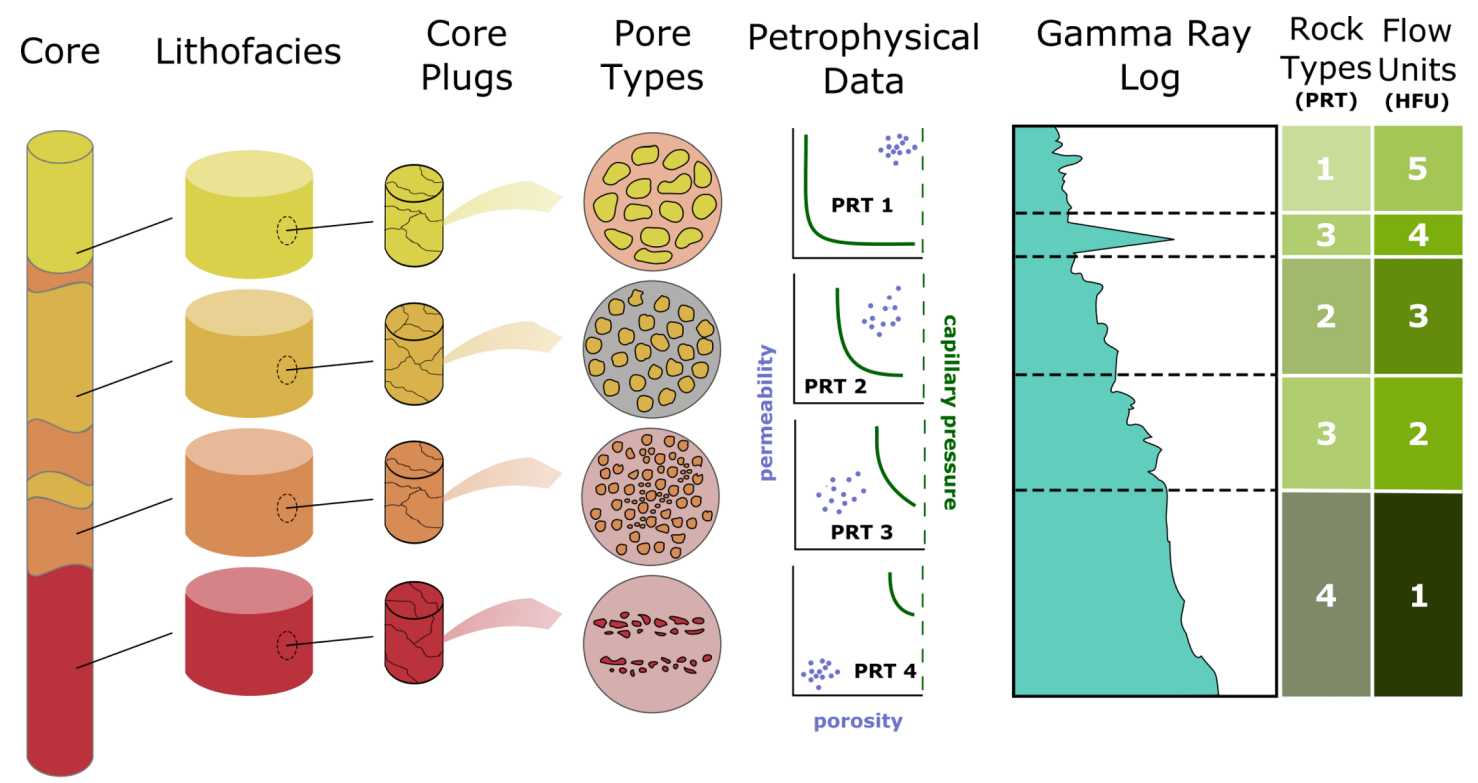

Fig. 1. Summarized process of petrophysical rock typing that relates flow properties to geologic features by harmonizing core description with lithofacies, pore types, rock-fluid data, well logs, rock types, and flow units. Modified from Gunter et al. (2012).

MATLAB $^{\circledR}$ which is also executable from GNU Octave. Secondly, we introduce an additional slope-based stairplot for the unidimensional segmentation of layers, which interprets the boundary between layers based on the $k / \phi$ ratio (reservoir process speed) and classifies the flow zones accordingly.

The paper is organized as follows: We first review the main graphical methods currently used in formation evaluation. Next, we introduce the general methodology and the specific tools included in GAWPS. Finally, we present its capabilities and validate and verify our implementation using specific benchmark models available in the literature. We also discuss the usage and applicability of the tools to various other cases.

\section{Background}

Fig. 1 outlines the general process of petrophysical rock typing from core sampling to final identification of flow units. A series of $\log$ profiles are commonly reported, providing an overview of the stratigraphy along a given wellbore, such as the gamma ray signal represented in the figure. However, a full understanding of production mechanisms can only be reached after thorough correlation between mineralogy, depth-wise layering, and seismic attributes, along with close examination of secondary variables, such as reservoir quality index, productivity potential, and heterogeneity level.

The whole process of reservoir characterization can be divided into stages. A few authors who have focused specifically on the petrophysical rock typing integration, provided four major stages fashioned under PIPM (Newsham and Rushing, 2001). The objectives of the different stages are as follows:

- Stage I (geologic assessment): assess geological features and define the architecture and geometry at the large scale.

- Stage II (petrophysical evaluation): describe the rock and fluid systems at the pore scale.

- Stage III (formation evaluation): integrate Stages I and II and describe the reservoir by using upscaling techniques.

- Stage IV (reservoir modeling): calibrate the geological and petrophysical models delivered by the previous stages and construct both two-dimensional (2D) and threedimensional (3D) models for wellbores and reservoir.

Fig. 2 is a reduced diagram of PIPM presented by (Rushing and Newsham, 2001), in which we emphasize only the third stage and highlight where the graphical methods come in as a fourth step in this stage. Although this process diagram is reasonable to comply with our objectives, an updated holistic version is recommended (Gunter et al., 2018).

\subsection{Flow units and statistics}

The hydraulic flow unit (HFU) is a concept intended to represent property variability in a facies model. Amaefule et al. (1993) define a HFU as "the representative elementary volume of total reservoir rock within which geological and petrophysical properties that may affect fluid flow are internally consistent and predictably different from other rock volumes." However, HFUs have a wide spectrum of application, and as discussed by Cannon (2015), there is a semantic variability in what an HFU refers to:

- to a geologist: a definable 3D facies object, such as a fluvial channel or a carbonate shoal;

- to a petrophysicist: a 2D correlatable zone with similar petrophysical properties; and

- to a reservoir engineer: a 3D reservoir layer that has a consistent dynamic response in the reservoir simulator.

To a reservoir modeller, however, an HFU is all these things.

From a geological viewpoint, there is a subtle distinction between facies and flow units. They can match, but this is not true for all cases, e.g., as illustrated by the petroleum system depicted in Fig. 3. 


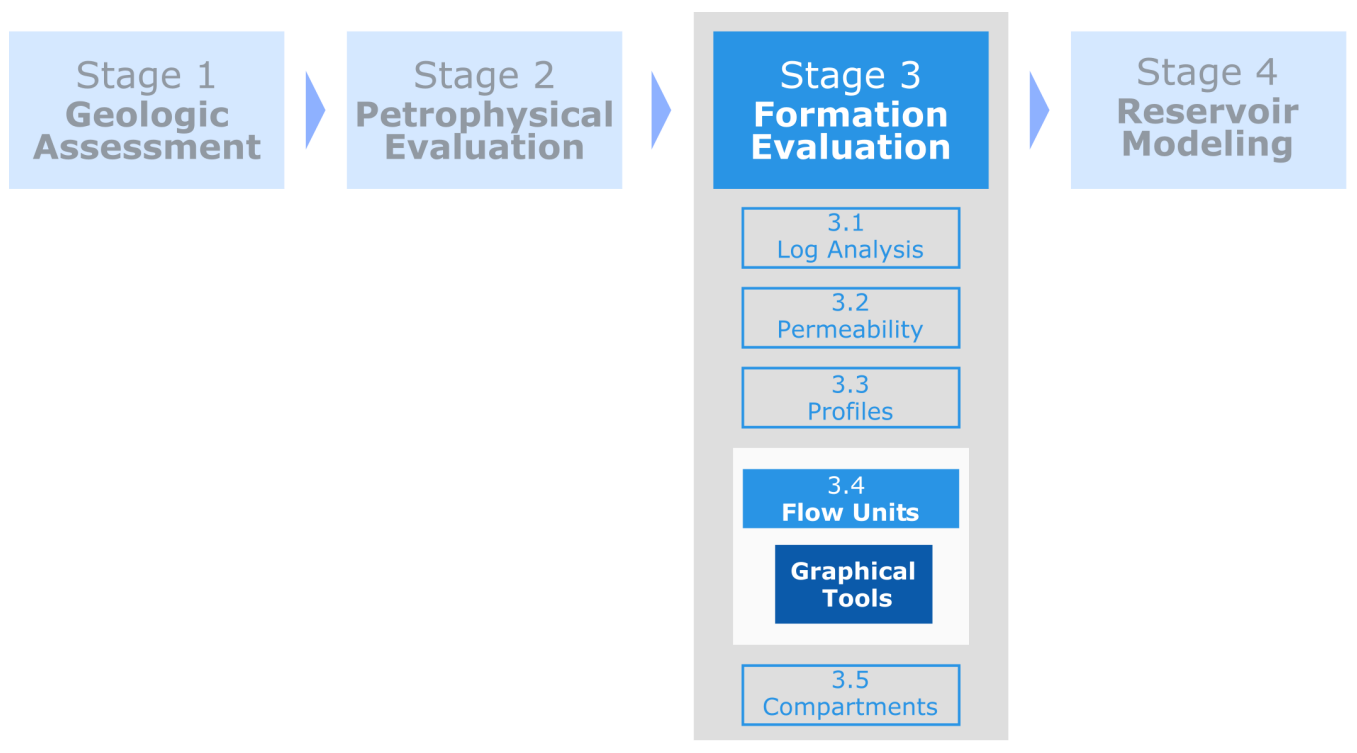

Fig. 2. Stages of PIPM. Stage III is expanded to show where the graphical tools appear as resources for flow unit analysis. Adapted from Rushing and Newsham (2001).

\section{Facies}

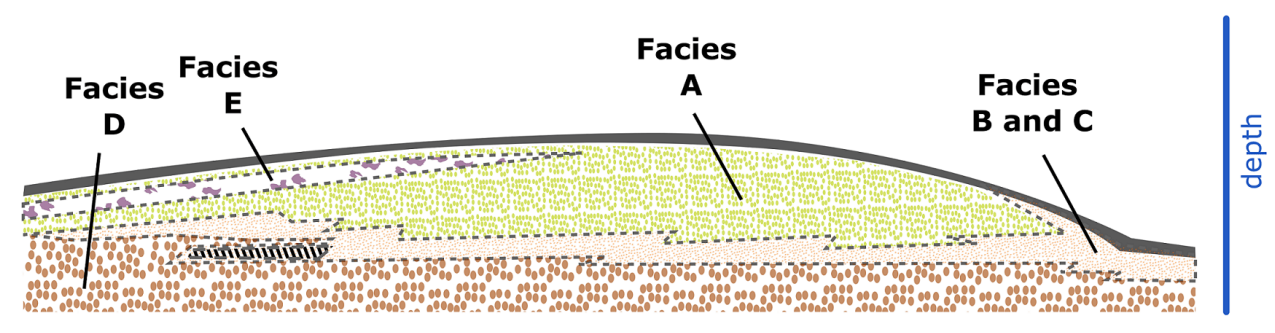

\section{Flow Units}

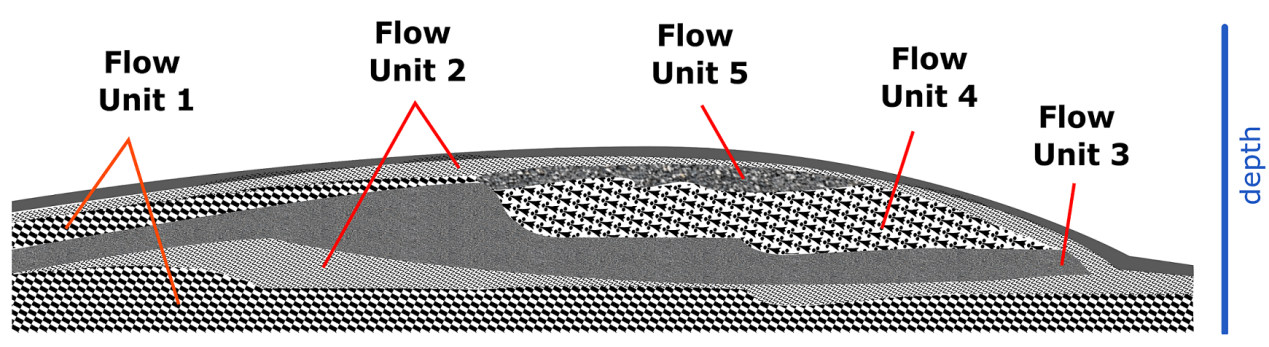

Fig. 3. Cross section of a stratigraphic sequence of facies (top) and respective flow units (bottom). This scheme shows that, due to the variable character of rock and flow properties, flow units and facies may have cover different domains, thereby resulting in unmatched boundaries. Facies A, for instance, is composed not only by a single unit, but by flow units 3, 4, and 5. Adapted from Hearn et al. (1984) and Ebanks Jr. et al. (1992).

From a statistical viewpoint, HFUs are volumes endowed with a rich convolution of textural and mineralogical factors, such as grain size, sorting, and pore throat radius, that have low variability. In other words, if the petrophysical properties across several flow units could be merged into a single variable, this variable would exhibit a probable behavior similar to that of a multimodal statistical distribution, where portions nearby a local maximum would represent the inner portion of a flow unit and regions close to valleys would represent boundaries of communicability (Abbaszadeh et al., 1996).

\subsection{Standard graphical methods}

There is a large class of methods used to identify flow units in oilfields. Many of these methods are inherently statistical, either linked to histogram analysis and probability plots (Mahjour et al., 2016) or to linear regression and clustering (Oliveira et al., 2016, 2020). To some extent, the quantitative profiles we review next, henceforth termed standard graphical methods, have the same intrinsic connection with statistics because they seek to single out the relationship among the main petrophysical properties used in reservoir modelling. 
Winland Plot

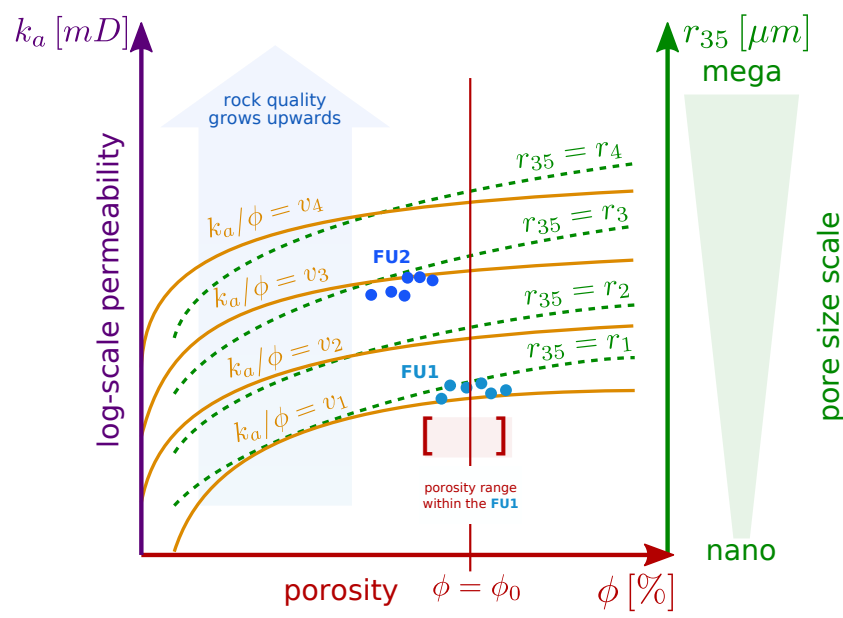

Fig. 4. Winland plot from two perspectives. When exhibited as $\log$-scale air permeability $\left(k_{a}\right)$ versus porosity $(\phi)$, isolines of constant $k_{a} / \phi$ (dark yellow) indicate bounds of rock quality. In this example, we single out four contours with values $v_{1}, \ldots, v_{4}$. The rock quality level grows upward. On the other hand, when exhibited as pore size aperture $\left(r_{35}\right)$ versus porosity, isolines of constant $r_{35}$ (called $r_{35}$ ports) are obtained (green). Four contours with values $r_{1}, \ldots, r_{4}$ are shown. Flow units are then detectable where measured data points form clusters around the isolines (blue). Here, FU2 is a flow unit of superior quality relative to FU1.

\subsubsection{Winland plot}

The Winland plot (Kolodzie Jr., 1980; Pittman, 1992) is a semi-log crossplot of air permeability $k_{a}[\mathrm{mD}]$ versus porosity $\phi[\%]$, in which isolines represent contours of the average pore throat radius (micrometers) taken from measurements read by capillary pressure tests at the $35^{\text {th }}$ percentile of mercury saturation. The isolines, also referred to as $r_{35}$ ports, can be computed from the empirical Winland's equation

$$
\log \left(r_{35}\right)=0.732+0.588 \log \left(k_{a}\right)-0.864 \log (\phi)
$$

or from other equations correlating porosity and permeability. This way, each intermediary area enclosed by a pair of isolines corresponds to a similar pore type. An usual pore throat classification based on the radius $r_{35}$ is given as follows:

- mega-porous: $r_{35}>10 \mu \mathrm{m}$;

- macro-porous: $2.5 \mu \mathrm{m} \leq r_{35} \leq 10 \mu \mathrm{m}$;

- meso-porous: $0.5 \mu \mathrm{m} \leq r_{35} \leq 2.5 \mu \mathrm{m}$;

- micro-porous: $0.2 \mu \mathrm{m} \leq r_{35} \leq 0.5 \mu \mathrm{m}$;

- nano-porous: $r_{35}<0.2 \mu \mathrm{m}$.

The Winland plot can have a dual axis to show the relationship between $k_{a}, \phi$, and the aperture size $r_{35}$. Flow units are recognized through clusters of data points whose quality is higher or lower depending on their position relative to an isoline. Hypothetically, as a cluster gets closer to an isoline, a rock volume with similar rock quality becomes identifiable, since $r_{35}$ and $k_{a} / \phi$ assume a constant value. Fig. 4 illustrates a Winland plot with both perspectives.

\section{Classic Lorenz Plot}

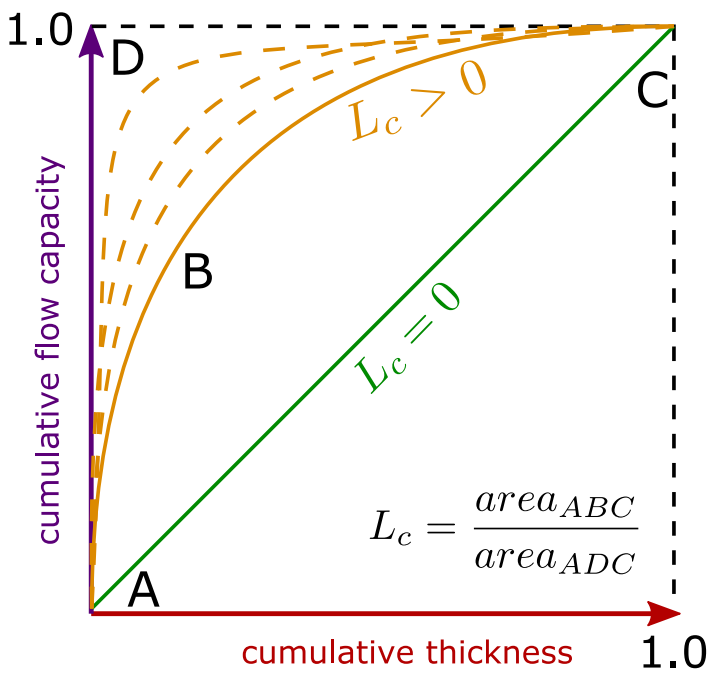

Fig. 5. Illustration of the classic Lorenz plot: the behavior of a unique curve plotted from cumulative flow capacity $\left(F_{m}\right)$ versus cumulative thickness $\left(H_{m}\right)$ describes the level of heterogeneity of the stratigraphic column.

\subsubsection{The classic Lorenz plot (CLP)}

The classic Lorenz plot measures the permeability heterogeneity across the layers of a reservoir. For a given wellbore having $n$ layers, each with thickness $h_{i}$ and permeability $k_{i}$, CLP is a curve of the cumulative flow capacity $F_{m}$ versus the cumulative thickness $H_{m}$, respectively computed as

$$
F_{m}=\frac{\sum_{i=1}^{m} k_{i} h_{i}}{\sum_{i=1}^{n} k_{i} h_{i}}, \quad H_{m}=\frac{\sum_{i=1}^{m} h_{i}}{\sum_{i=1}^{n} h_{i}}, \quad m=1, \ldots, n
$$

One readily verifies that both quantities have unity as an upper bound so that the generated curve divides a square into two areas as depicted in Fig. 5.

The Lorenz coefficient, defined as twice the area bounded above by the $\left(F_{m}, H_{m}\right)$ curve and below by the the 45 -degree bisector line $\mathrm{AC}$, measures the level of heterogeneity depthwise. For a homogeneous medium, all $k_{i}$ values are equal and the Lorenz curve degenerates to the bisector line $\mathrm{AC}$ and $L_{c}=0$. As heterogeneity increases, the curve $\mathrm{ABC}$ shifts towards $\mathrm{D}$ and $L_{c} \rightarrow 1$. Typical values lie in the range $[0.2,0.6]$ (Fanchi, 2010). CLP and $L_{c}$ are adapted versions of the Lorenz curve and Gini coefficient, concepts applied in economics to assess imbalances of wealth distribution. The $L_{c}$ coefficient is somewhat more difficult to compute because of permeability ordering, and one can instead use alternative measures like the Dykstra-Parsons or the variation coefficient (Craig, 1971).

\subsubsection{The stratigraphic modified Lorenz plot (SMLP)}

SMLP is a curve of the percentage flow capacity versus the percentage storage capacity, ordered according to the stratigraphic sequence (Gunter et al., 1997a). Flow capacity and storage capacity are defined, respectively, by the products $k_{i} h_{i}$ and $\phi_{i} h_{i}$ for the $i$-th layer of the reservoir. When they 


\section{Stratigraphic Modified Lorenz Plot}

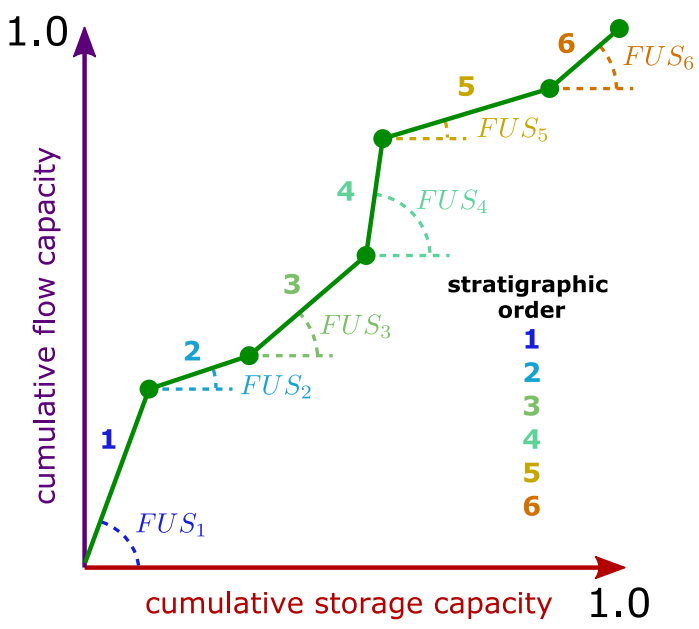

\section{Modified Lorenz Plot}

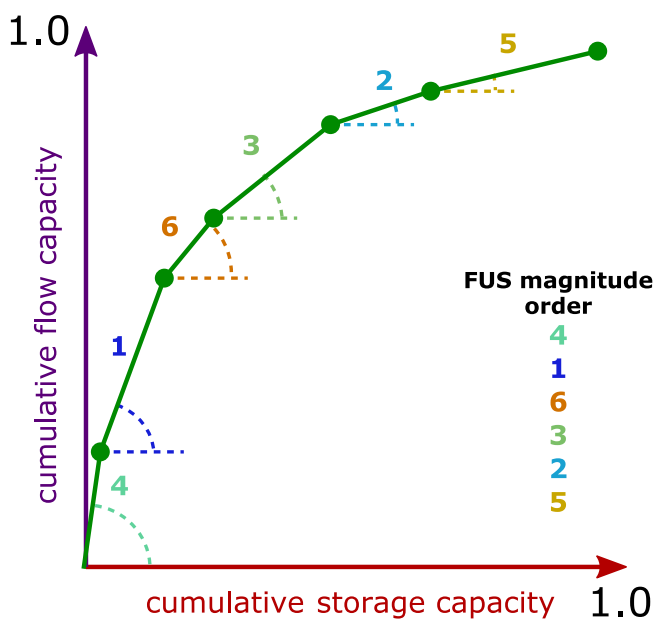

Fig. 6. Comprehensive illustration of SMLP (left) and MLP (right). The former is a plot of cumulative flow capacity $\left(F_{m}\right)$ versus cumulative storage capacity $\left(S_{m}\right)$. A piecewise linear function is obtained when joining the individual segments whose inclinations are exactly the FUS. In this example, there are six distinct flow units. Steeper slopes mean higher speed zones. The latter plot is obtained after breaking down the SMLP and reordering the segments (flow units) according to decreasing FUS's magnitude. In summary, SMLP honors the stratigraphic order, whereas MLP focuses on a qualitative order.

are individually summed, we find the total capacities of the reservoir. The percent storage capacity is given by

$$
S_{m}=\frac{\sum_{i=1}^{m} \phi_{i} h_{i}}{\sum_{i=1}^{n} \phi_{i} h_{i}}, \quad m=1, \ldots, n
$$

Hence, SMLP results from the plot of $F_{m}$ versus $S_{m}$. A parallelism can be traced between $F_{m}$ (or $S_{m}$ ) and a modified version of Stile's method (Dake, 2001), an earlier technique implemented for flooding order in direct calculations of oil recovery following water breakthrough in non-communicating layers.

SMLP has a multifunctional interpretation. Firstly, the curve behaves as a piecewise linear function. Each segment relates to the inner portion of a HFU and the inflection points are HFU boundaries. Secondly, the slope of each segment is the ratio between the local percentage flow capacity and the local percentage storage capacity, which is defined as the flow unit speed $(F U S)$. Without losing generality, we can write $F U S_{i}=[k h \%]_{i} /[\phi h \%]_{i}$, where the numerator and the denominator are local values of $F_{m}$ and $S_{m}$, respectively. In fact, FUS is a local expression of the reservoir speed process (RPS), here denoted by $v=k / \phi$. Because the dimension of $v$ is the same as permeability and $F U S$, their similarity with the instantaneous velocity from physics $(v=d s / d t)$ is quite clear. Thirdly, the length of each segment can be seen as an indication of how thick is the flow unit.

Fig. 6 (at left) is a comprehensive illustration of the SMLP. Therein we observe the existence of six flow units with varying $F U S$ values. A direct interpretation is that higher slopes lead to higher speed zones. Additionally, SMLP seeks to honor the stratigraphic ordering of the flow units. In this illustration, $F U S_{1}>F U S_{3}$, for example, and flow unit 3 is deeper than flow unit 1.

\subsubsection{The modified Lorenz plot (MLP)}

MPL is calculated in a similar way as SMLP but is similar to CLP in appearance. Basically, MLP is obtained by cutting the SMLP curve exactly at its inflection points (flow unit boundaries) and rearranging the segments in decreasing order of FUS (Gunter et al., 1997b). This way, MLP breaks down the stratigraphic order and favors the qualitative order. In Fig. 6, MLP relative to the previous SMLP is illustrated on the right. As observed, the stratigraphic order $\{1,2,3,4,5,6\}$ is sorted to $\{4,1,6,3,2,5\}$ because $F U S_{4}>F U S_{1}>\cdots>F U S_{5}$.

\subsubsection{The normalized cumulative flow capacity plot (NCFCP)}

This plot is not shown here but is equivalent to SMLP, except for the abscissa axis, which is taken to be the true depth (Fanchi, 2010). Normalized naturally means limited by 1.0 and the ordinate axis of the NCFCP is $F_{m}$. Usually, the curves of SMLP and NCFCP overlap, but they might have minor deviations in some cases.

\subsubsection{The normalized cumulative RQI plot (NCRQIP)}

The normalized cumulative reservoir quality index plot is the curve obtained when plotting the true depth $h$ versus

$$
R Q I_{m}=\frac{\sum_{i=1}^{m} R Q I_{i}}{\sum_{i=1}^{n} R Q I_{i}}, \quad m=1, \ldots, n
$$

where the reservoir quality index $(R Q I)$ is defined as $R Q I=$ $0.0314 \sqrt{k_{i} / \phi_{i}}$ (Amaefule et al., 1993) (The conversion factor to millidarcies, 0.0314 , can be cancelled). This plot was established to recognize flow units and speed zones by 
Multimodal log-normal FZI distribution

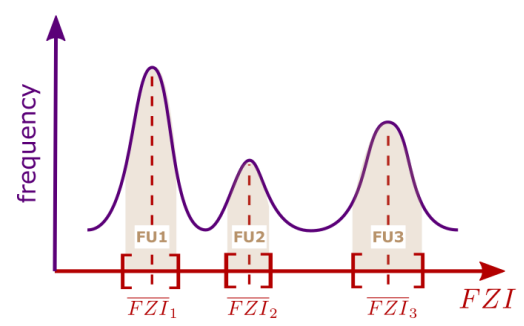

Normal distribution

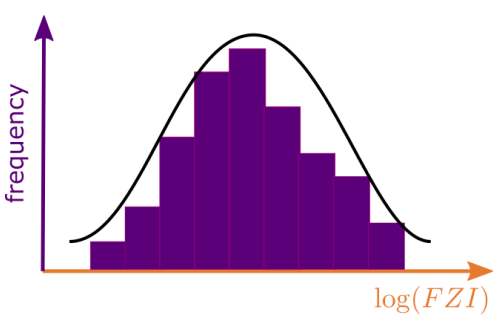

Normal Probability Plot

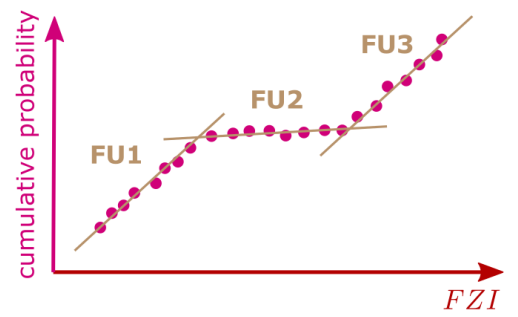

Fig. 7. Comprehensive illustration of the normal probability plot (NPP): $F Z I$ is a multimodal log-normal distribution whose peaks are average values $\left(\overline{F Z I_{i}}\right)$ that characterize mineralogical aspects of individual flow units (left). Then, the histogram of $\log (F Z I)$ behaves as a normal distribution (middle), from which the probability plot decouples the original superimposed modes that are identified through straight lines (right). In ultimate analysis, the data points orbit around average values slightly deviating from normality, so that each near-collinearity condition indicates a flow unit domain.

observing the slope of the segments that compose a piecewise linear function (Siddiqui et al., 2003). In fact, it acts like FUS does at SMLP. Because NCRQIP originally places the depth along the ordinate axis, its interpretation occurs under an inverted perspective in contrast to SMLP. While higher slopes indicate higher flow zones for SMLP, steeper slopes for NCRQIP mean lower flow zones, instead.

\subsubsection{The normal probability plot (NPP)}

This plot is commonly employed in general statistical analysis to identify data sets that follow a normal distribution. Its application in HFU identification relies on the flow zone indicator $(F Z I)$, defined as $F Z I=R Q I / \phi_{z}$, where $\phi_{z}=\phi /(1-\phi)$ is called the pore-to-matrix ratio (Amaefule et al., 1993).

It is known that $F Z I$ has a log-normal multimodal distribution (Abbaszadeh et al., 1996). Each peak represents an average value that establishes a flow unit. The histogram of $\log (F Z I)$ shows a normal distribution formed by an $N$-mode superposition. When mapped onto a probability plot, $N$ straight lines can be visualized, which represent $N$ coherent groups of mineralogy, namely the flow units. In other words, NPP provides a desuperposition that can be refined through other separation techniques, such as constrained clustering (Oliveira et al., 2020). It is interesting to observe, however, that the slopes of these straight lines at the NPP are deviations of normality and, at a first glance, they have nothing to do with $F U S$. Relevant information provided by NPP is the number of possible HFUs detectable for the well under analysis. Histogram analysis and NPP complement statistical verification in studies encompassing oilfields located all over the world (Svirsky et al., 2004; Zhou et al., 2014; Hatampour et al., 2015). Fig. 7 illustrates the relationship between $F Z I$ and probability plots.

\section{Methods}

\subsection{The MRST framework}

The MRST (Lie, 2019) is a free, open-source extension of MATLAB $^{\circledR}$, a proprietary multi-paradigm programming language and numerical computing environment. MRST is designed as a toolbox consisting of a great variety of routines to assist rapid prototyping and testing of new models, computational algorithms, and workflows for modelling and simulating flow processes in porous media. The software is organized as a core module offering basic functionality such as plotting routines, routines for reading and processing industrystandard data formats in reservoir engineering, methods for unit conversion, data structures and grid generators to represent reservoir geometry, and data objects to represent petrophysical properties, and drive mechanisms like gravity, wells, volumetric source terms, and boundary conditions (e.g., aquifers). The core module also implements a library for automatic differentiation.

The major part of the software consists of add-on modules that extend the basic functionality with discretizations, solvers, models of flow and mechanics, as well as a wide variety of specific simulator- and workflow tools. In particular, MRST has an object-oriented simulator framework based on automatic differentiation, which is designed to simplify the process of developing new (and fully differentiable) simulators, but which also offers enough functionality to enable fully-fledged reservoir simulations.

\subsection{Code design}

Fig. 8 shows a schematic view of how GAWPS adheres to MRST as an add-on module. Only a few objects were highlighted to show how GAWPS' capabilities require functionality from MRST's core module as well as readable reservoir models, represented in the ECLIPSE $^{\circledR}$ input format (or, in principle, in some other format that the standard input routines MRST can read and process).

GAWPS is designed using elementary functionality of MRST. Fig. 9 is a diagram that expands GAWPS objects and shows their intercommunication. The module can be arranged as a set of methods. In particular, the function resmodel provides a pipeline to read several ECLIPSE ${ }^{\circledR}$ deck inputs.

GAWPS reuses basic functionality from MATLAB and MRST for data analysis and visualization. Because all the analyses in GAWPS are performed on one-dimensional profiles only, the computational costs scale with the number of completed layers (and the number of wells) and not with the number of cells in a model. GAWPS thus offers good performance for medium-sized to large models. The main limiting factor lies in that well traces cannot be read directly 


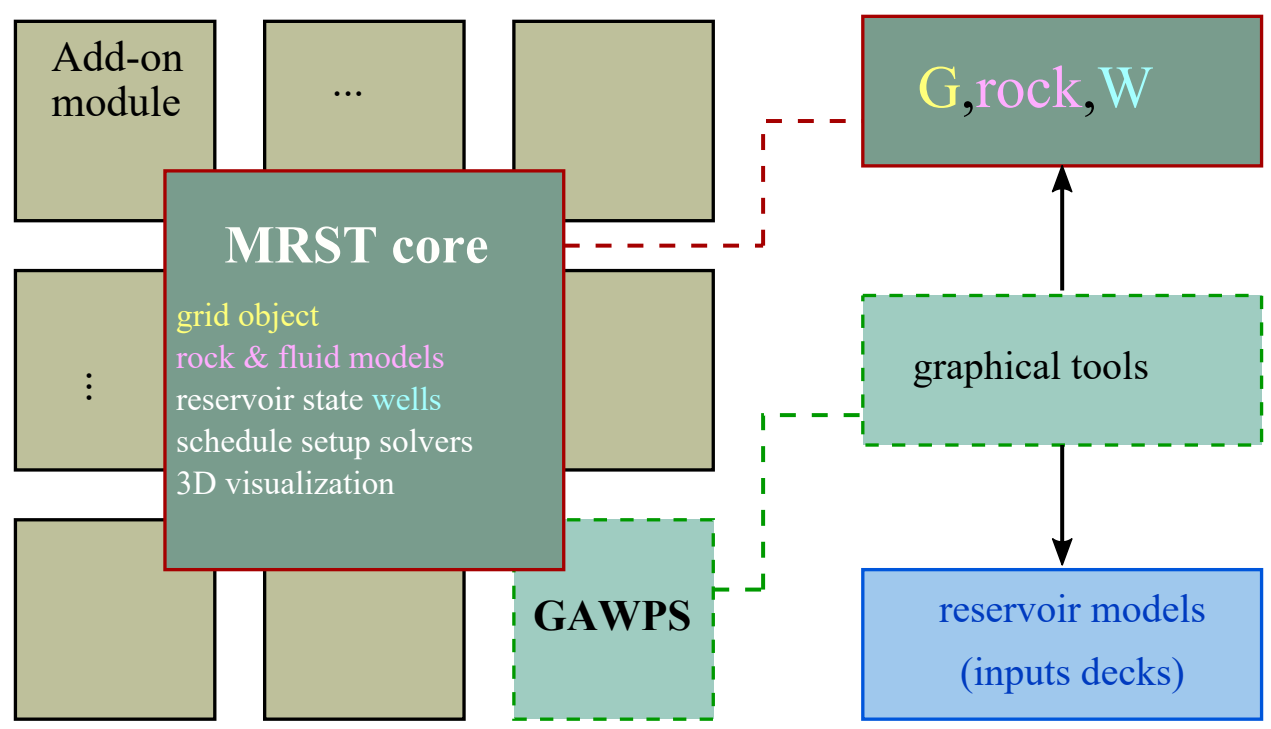

Fig. 8. Schematic view of MRST framework highlighting core structures of the software, add-on modules, and GAWPS' communication with objects related to basic functionality and reservoir models.

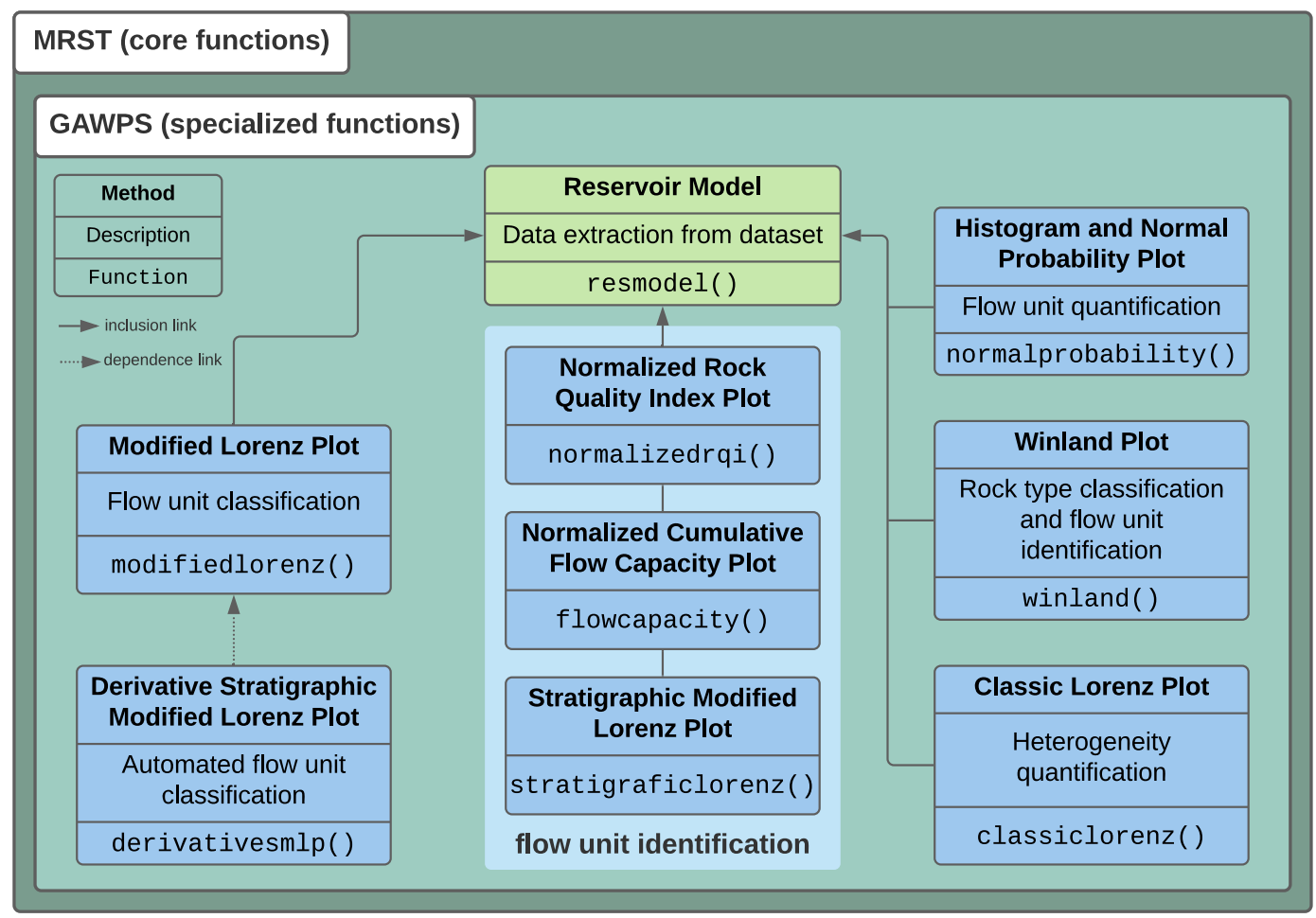

Fig. 9. Description of the main computational routines implemented in the GAWPS module.

from an input file, but must be extracted from a full 3D model. MRST's routines for input reading and preprocessing 3D models are efficient but cannot compete with dedicated routines implemented on high-performance hardware for models with multi-million cells. The 3D visualization in MATLAB can also be slow compared to highly optimized rendering codes on dedicated hardware. However, we have not experienced any of these factors as limitations for the model sizes shown herein, which are representative of real field cases.

\subsection{Speed zone classification}

As shown in Fig. 6, SMLP and MLP provide profiles wherein slope variations relate to lower or higher FUSs that will determine distinct classifications. In the literature, we find three major classes of flow units: i) seals (or barriers) are zones that impede flow and isolate zones as individual pressure compartments; ii) baffles are zones that block or divert the flow; and iii) normal units are zones that facilitate fluid flow motion. However, the presence of terms such as "super-permeable units", "super-K units", "speed zone units", 
and others lead us to conclude that flow unit classification in terms of FUS is somehow subjective (Cunningham et al., 2009; Rahimpour-Bonab et al., 2014). In particular, the concept of "baffle" may be so sensitive to lithology that "strong baffle" or "weak baffle" become conceivable classes, just as baffle quality indicator or baffle continuity indicator are plausible choices to classify flow units from stratigraphy (Benham et al., 2018).

Here, we adopt the following classification for well intervals

$$
\alpha(F U S)= \begin{cases}\text { barrier (B), } & \text { if } 0.0 \leq F U S \leq 0.1 \\ \text { strong baffle }(\mathrm{SB}), & \text { if } 0.1<F U S \leq 0.5 \\ \text { weak baffle (WB), } & \text { if } 0.5<F U S \leq 1.0 \\ \text { normal unit (NU), } & \text { if } 1.0<F U S<\infty\end{cases}
$$

This way, we consider as limit of "normal unit" the condition when $F U S$ surpasses 1.0, i.e., $d F_{m} \propto d H_{m}$ in terms of local derivative. Evidently, barriers are zero-slope zones, whereas baffles are intermediary conditions with slopes below unity. The so-called "super-K units" would be assignable to intervals where $F U S \gg 1.0$. However, the user can customize the limits for FUS inside the computational routine and redefine $\alpha$ to narrow or stretch the ranges.

\subsection{The derivative stratigraphic modified Lorenz plot (DSMLP)}

To enable an automated process for classifying speed zone units, we propose to consider the derivative of SMLP as a new plot. As discussed in Subsection 2.2.3, SMLP is a piecewise linear curve whose junction knots represent frontiers separating two stratigraphic groups. Because the knots are inflection points, we can compute the derivative of SMLP and look for jumps that will mark local changes of speed. DSMLP is a stair-like plot that gives us major information: the constant-value plateaus stand for local values of RPS ; upscaled plateaus define a speed zone that should be classified from its corresponding $F U S$ value. Hence, FUS is an average-based indicator that requires a kind of clustering of groups of localized speeds per reservoir layer. In GAWPS, DSMLP is obtained by applying the gradient function on SMLP. Resulting plots will be shown later.

\subsection{Depth-wise speed unit clustering}

DSMLP is useful to detect inflection points, but should be upscaled to produce depth intervals suitable for characterization. We undertake a $k$-means clustering to group proximal values from the DSMLP plot into a mean value that will be used as input to the function $\alpha$ to classify depth layers as defined in Eq. (5).

Such depth-wise speed unit clustering is explained as follows. Let us suppose that $W(i, j)=\left\{w_{(i, j, z)}\right\}_{z=1}^{n}$ represents a discrete well with surface coordinates $(i, j)$, formed by $n$ grid cells stacked depth-wise, and let $R=\left\{R_{z}\right\}_{z=1}^{n}$, where $R_{z}=\operatorname{RPS}\left(w_{(i, j, z)}\right)=\operatorname{RPS}\left(h_{z}\right)$. Because $w_{(i, j, z)}$ and $h_{z}$ have a one-to-one correspondence, it holds $H=\left\{h_{z}\right\}_{z=1}^{n}$, so that we can solve the following partitioning problem over the feature set $(R, H)$ : Find $n_{p}$ nonempty partitions $\left(R^{(1)}, H^{(1)}\right),\left(R^{(2)}, H^{(2)}\right), \ldots,\left(R^{\left(n_{p}\right)}, H^{\left(n_{p}\right)}\right)$, given the initializa- tion set $\left(R^{0}, H^{0}\right)$, where $R^{0}=\left\{\llbracket R P S \rrbracket_{p}\right\}_{p=1}^{n_{p}}$ is the set of the $n_{p}$ largest jumps $\llbracket R P S \rrbracket$ observed in the DSMLP plot, and $H^{0}$ their corresponding depths.

A $k$-means clustering with a fixed initial seed (matrix of centroids) is proposed here. The choice $\left(R^{0}, H^{0}\right)$ just stated enables us to reach a reasonable solution that respects linear boundaries among the generated clusters. Such boundaries are equivalent to the frontiers of the speed zones. The user can choose the number of $n_{p}$ input groups freely based on a previous screening of the RPS jumps. Once $n_{p}$ is determined, the code will assign

$$
F U S_{p}=\frac{1}{\# R^{(p)}} \sum_{m=1}^{\# R^{(p)}} \operatorname{RPS}\left[w_{(i, j, m)}\right]
$$

where $\# R^{(p)}$ is the number of values belonging to $R^{(p)}$. Hence, $\alpha\left(R^{(p)}\right)=\alpha\left(F U S_{p}\right)$, thus defining that all cells $w_{(i, j, \cdot)}$ associated to the depths corresponding to the $R^{(p)}$ values will be ascribed with the same class. It turns out that the classification of speed units along $W(i, j)$ is unsupervised and average-based. Besides, it will vary in the number of units depending on the interpretation of the RPS jumps over the well column. Mathematically, the clusters can be viewed as points on the plane $R P S$ versus depth.

\section{Usage and applications}

This section outlines how the code can be used to analyse different reservoir models with emphasis on benchmarks from the literature. To keep the demonstration short, clear, and broad, each graphical method from GAWPS was applied to only two sample wells (labels: $W_{1}, W_{2}$ ), chosen randomly to avoid cluttered plots. Detailed interpretation along each well is not the main purpose of this section, because judgments can vary from user to user.

However, some interpretations are included where noteworthy opinions were deemed necessary to clarify the interpretation for the reader, but we emphasize that our interpretations not take into account conciliation of $\log$ and core data, log analysis, or other relevant aspects that must be integrated. All tests here can be replicated by the user and some of them are modifiable depending on input settings. The applications demonstrated per dataset are summarized in Table 1.

\subsection{Dataset 1: SPE10 model}

The 10th SPE Comparative Solution Project (Christie and Blunt, 2001) was designed to compare upscaling approaches and predict waterflood performance. Model 2 from the benchmark is a regular Cartesian grid with approximate dimensions $366 \times 671 \times 52 \mathrm{~m}^{3}$.

The top 21 meters (35 layers) represent the Tarbert formation and the bottom 31 meters (50 layers) represent the Upper Ness. The cell size is approximately $6 \times 3 \times 0.6 \mathrm{~m}^{3}$ as depicted in Fig. 10. Two wells are placed in the middle of the reservoir to sample permeability and porosity values; the permeability is computed as the geometric mean of the lateral and vertical permeabilities.

Fig. 11 reports the Winland plot, SMLP, and MLP. In the Winland plot it is possible to verify the existence of blue points 

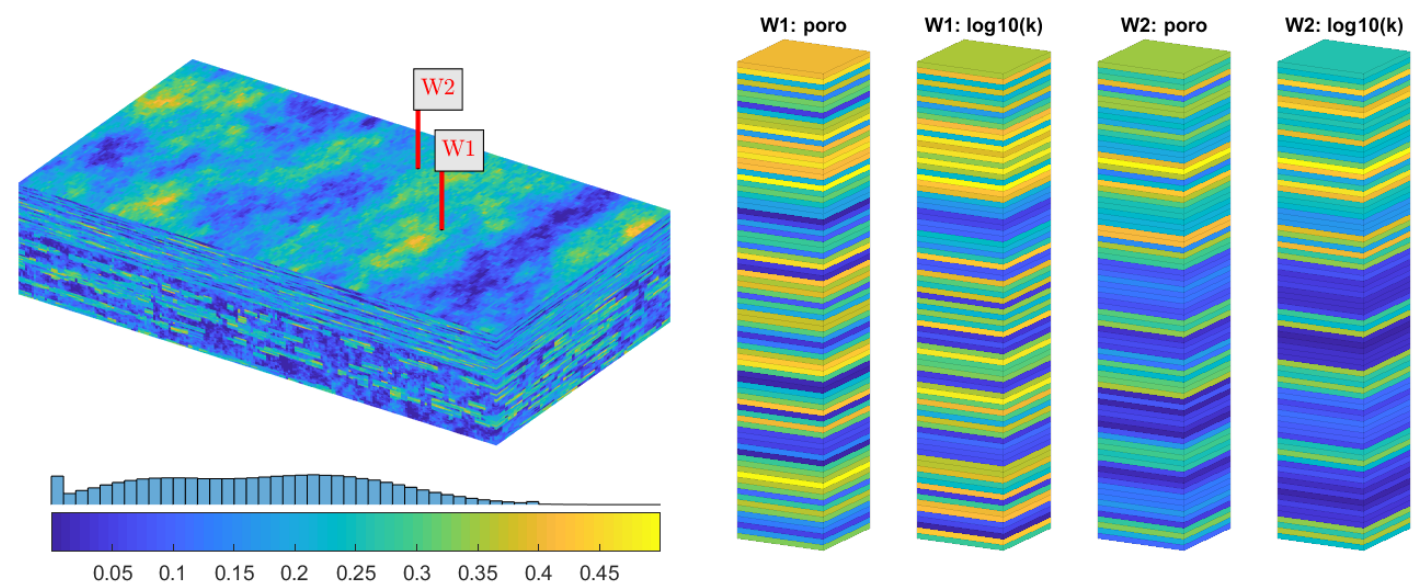

Fig. 10. 3D overview of the stratigraphy of Model 2 from the 10th SPE Comparative Solution Project (SPE10). We plotted the porosity for the full field as well as porosity and permeability traces along two study well heads located in the middle of the reservoir.
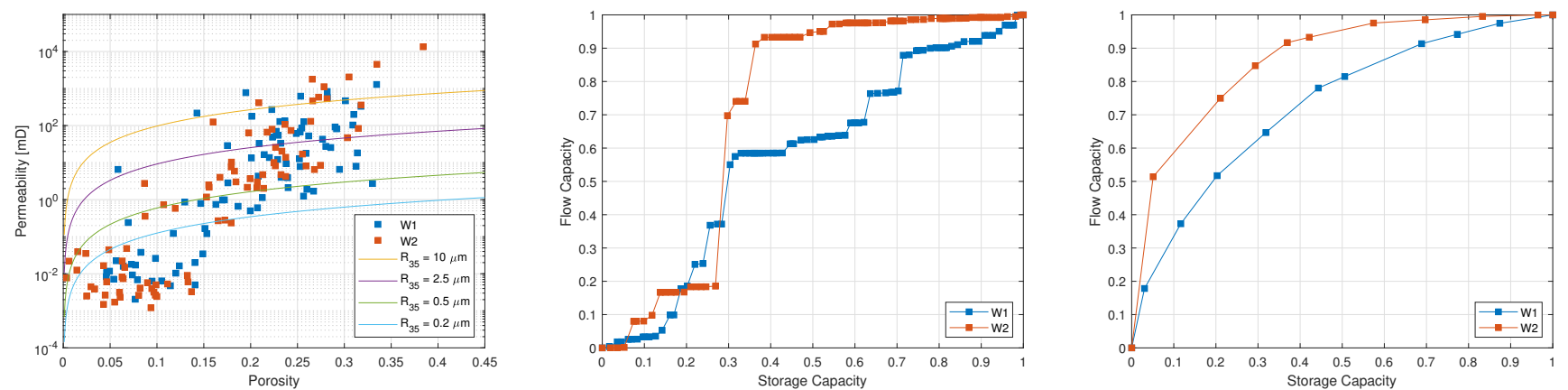

Fig. 11. Graphical methods applied to wells $W_{1}$ and $W_{2}$ for the SPE10 model: Winland plot (left), SMLP (middle), and MLP (right).

Table 1. Organized list of graphical tools presented per dataset in this paper. The choice of the plots is merely optional to keep the exposition short.

\begin{tabular}{ll}
\hline Model (dataset) & Graphical tools \\
\hline \multirow{3}{*}{ SPE10 } & WP \\
& SMLP, MLP \\
& NPP + histogram \\
& DSMLP + clustering map \\
& NCRQIP \\
UNISIM-I-D & SMLP, MLP \\
& NPP + histogram \\
& DSMLP + clustering map \\
UNISIM-II-D & CLP \\
& NCFCP \\
Norne & WP \\
Egg & NCFCP \\
\hline
\end{tabular}

$\left(W_{1}\right)$ clustered around the isoline $r_{35}=0.5 \mu \mathrm{m}$ and another cluster of red points $\left(W_{2}\right)$ that forms between the isolines $r_{35}=0.5 \mu \mathrm{m}$ and $r_{35}=2.5 \mu \mathrm{m}$, meaning that flow units with micro- to meso-porous traces are identifiable in both wells.

SMLP shows different number of slopes for each well, but a few behaviors are noticeable. For $W_{2}$, for instance, the steepest segment in the plot indicates a high speed zone at intermediary depths. Near the bottom, we see a long segment with small slope that may indicate a low-speed unit. On the other hand, in $W_{1}$, a succession of small jumps interspersed between longer small-slope segments point to more distributed units than $W_{2}$.

Information from MLP complements the previous analysis. The current plot considered ten flow units for each well, each separated by a slope change. That is why only ten points are marked each curve. However, more meticulous thresholds, leading to a greater number of flow units, could be used.

Fig. 12 depicts the individual NPP and histogram plots for each well. Both plots are created from visual modifications of the built-in NPP ${ }^{1}$ and histogram plots available in MATLAB ${ }^{\circledR}$. NPP is an additional technique that allow us to highlight flow units by tracing straight lines (see Fig. 7). However, this feature is not automatically implemented inside GAWPS, so that some manual work is required to trace such support lines. Histograms are often used for frequency analysis and accompany the NPP-

\footnotetext{
${ }^{1}$ This plot is rendered with using the normplot function, provided by the Statistics and Machine Learning Toolbox from MATLAB ${ }^{\circledR}$.
} 


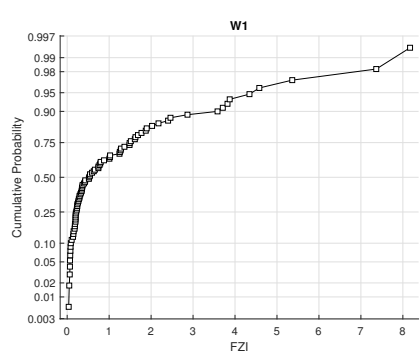

(a) NPP $\left(W_{1}\right)$

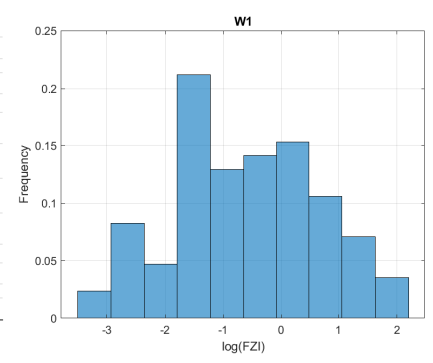

(b) Histogram $\left(W_{1}\right)$

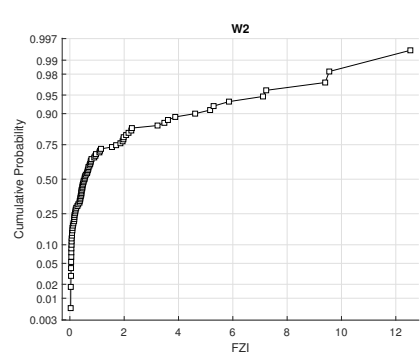

(c) NPP $\left(W_{2}\right)$

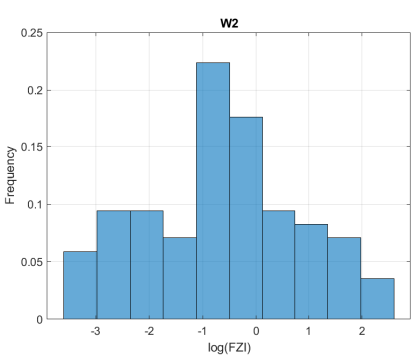

(d) Histogram $\left(W_{2}\right)$

Fig. 12. NPP and histogram plot applied to wells $W_{1}$ and $W_{2}$ from the SPE10 model.

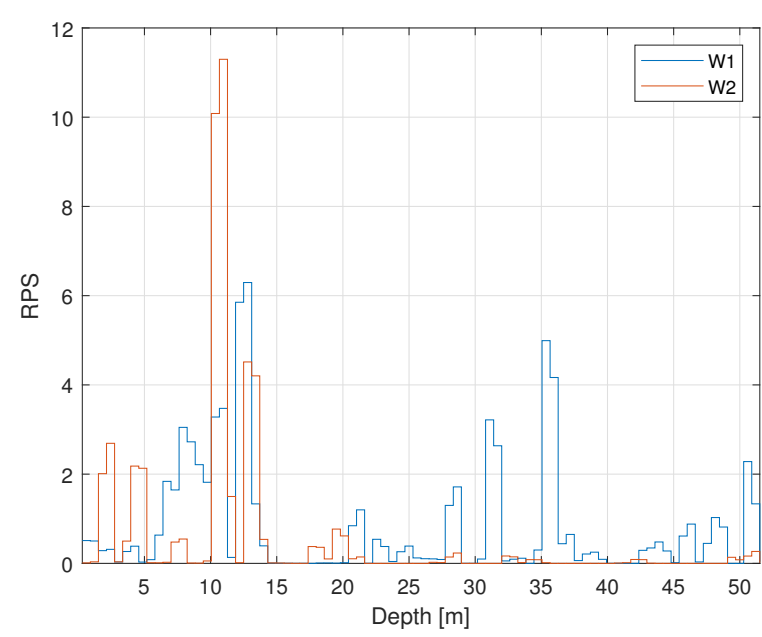

Fig. 13. DSMLP for the SPE10 model including the two sampled wells.

based visualization. In GAWPS, histogram plots are used to distribute the FZI over each well with fixed binning (10 groups by default).

Fig. 13 reproduces the DSMLP for both wells. As introduced in Subsection 3.4, DSMLP outlines the RPS locally along depth over the wellbore. Because RPS is a piecewise constant derivative, DSMLP can be drawn as a stair-like plot. Each jump marks out a flow unit boundary and the higher the plateau, the higher is the flow speed locally over the well. Although RPS is measured in permeability units, protruding spikes in the plot identify speed zones. Similar to spectral analysis, DSMLP can be interpreted as an "energy" locator. The current plot shows, for instance, that within the range 10$15 \mathrm{~m}, W_{2}$ crosses a normal unit with elevated speed. Because $R P S$ can vary abruptly, upscaling blobs of speed in a unique bundle is a way to achieve more consistent characterizations. For example, within $45-50 \mathrm{~m}$, speeds of the same magnitude are observed, which can be merged and give rise to a single unit.

Fig. 14 illustrates the final stage of classification provided by GAWPS applied to wells $W_{1}$ and $W_{2}$ over the SPE10 model: the RPS-clustering map, $F U S$ values, and speed zone classification $\alpha(F U S)$. The RPS-clustering map is provided by the function gscatter with modified appearance. It depicts the labelled RPS groups clustered through the $k$-means algorithm as explained in Subsection 3.5. In this example, ten clusters are defined, agreeing with the number of flow unit observed in MLP (frames (a) and (b)). This way, the sets $R^{(i)}, i=1,2, \ldots, 10$ correspond to $R P S$ values that are grossly "upscaled" when defining speed units. Black stems indicate the average RPS values for each cluster. The proper meaning of this map is obtained by transferring the cluster labels directly to wellbore views of $F U S$ values, which are the unit slopes that will be used as inputs to the function $\alpha$. As observed, the $F U S$-scale varies from well to well (frames (c) and (d)), so that $W_{1}$ and $W_{2}$ differ considerably in classification (frames (e) and (f)). In this example, $W_{1}$ has two ranges of normal units, one longer and one shorter, separated by baffles, whereas $W_{2}$ has a large zone classified as a barrier near the bottom.

\subsection{Dataset 2: UNISIM-I-D model}

UNISIM-I-D is a model for the Namorado Oil Field, Campos Basin, Brazil, formed by a grid with $81 \times 58 \times 20$ cells measuring $100 \times 100 \times 8[\mathrm{~m}]$ (Avansi and Schiozer, 2015). This benchmark was developed for exploitation strategy selection. Here, we used the deterministic dataset published by the Cepetro/Unicamp group. A 3D view of the permeability distribution is presented in the 3D view in Fig. 15.

In this case, we highlight the function of the normalized cumulative RQI plot (NCRQIP), because the other plots mentioned in Table 1 that were also applied to this case were just discussed for SPE10. NCRQIP enables us to identify quality ranges directly by looking at the depth axis. Fig. 16 depicts NCRQIP, SMLP, and MLP for two arbitrary wells chosen over the UNISIM-I-D model.

Approximately five units are inferred per well identified by straight line segments from NCRQIP and aided by MLP. For instance, marked slope changes are seen a little bit above 3,040 $\mathrm{m}$ and close to $3,050 \mathrm{~m}$ for $W_{1}$. For $W_{2}$, mild slope changes appear. As seen, the blue curve covers a depth range reaching a maximum of 3,080 $\mathrm{m}$, while the red curve goes further up to $3,140 \mathrm{~m}$, showing that wells cover distinct ranges. Moreover, unevenly spaced points single out varying layer thickness, which exists as a result of the grid's corner-point structure. In GAWPS, layer thicknesses are computed by averaging pillar heights.

Fig. 17 depicts the normal probability plot and histogram for the same wells in UNISIM-I-D. In this case, the number of flow units can be seen more easily by observing NPP because "broken lines" are perceptible. In $W_{2}$, for instance, a segment 

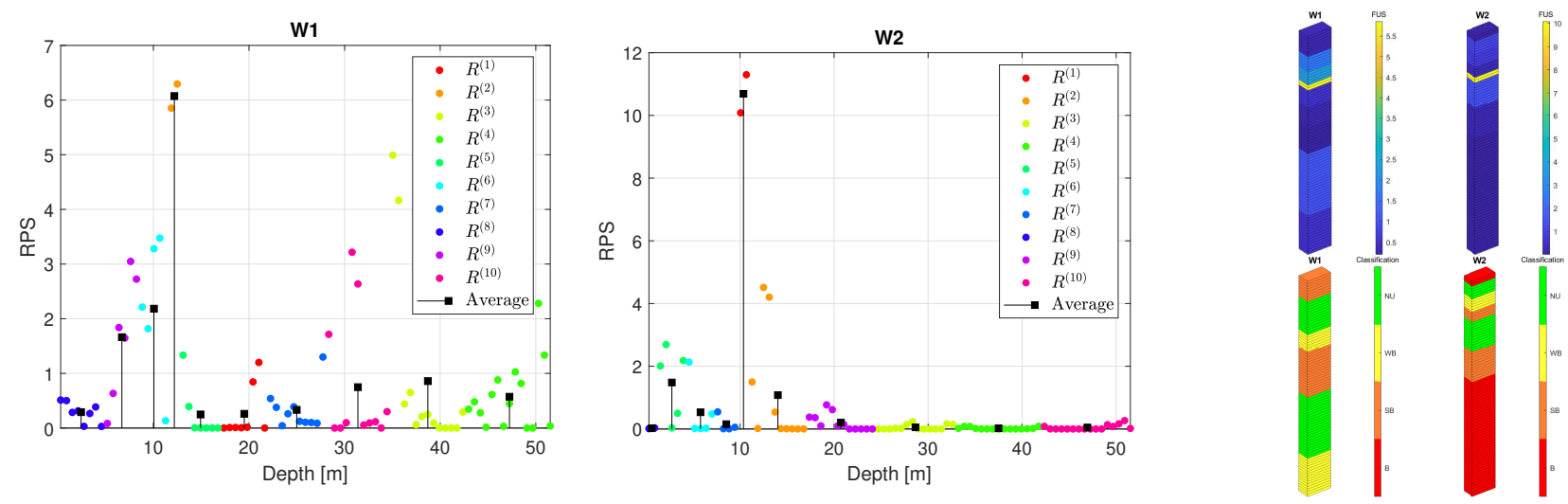

Fig. 14. RPS-clustering maps, FUS values, and speed zone classification $\alpha(F U S)$ for two wells over the SPE10 model.
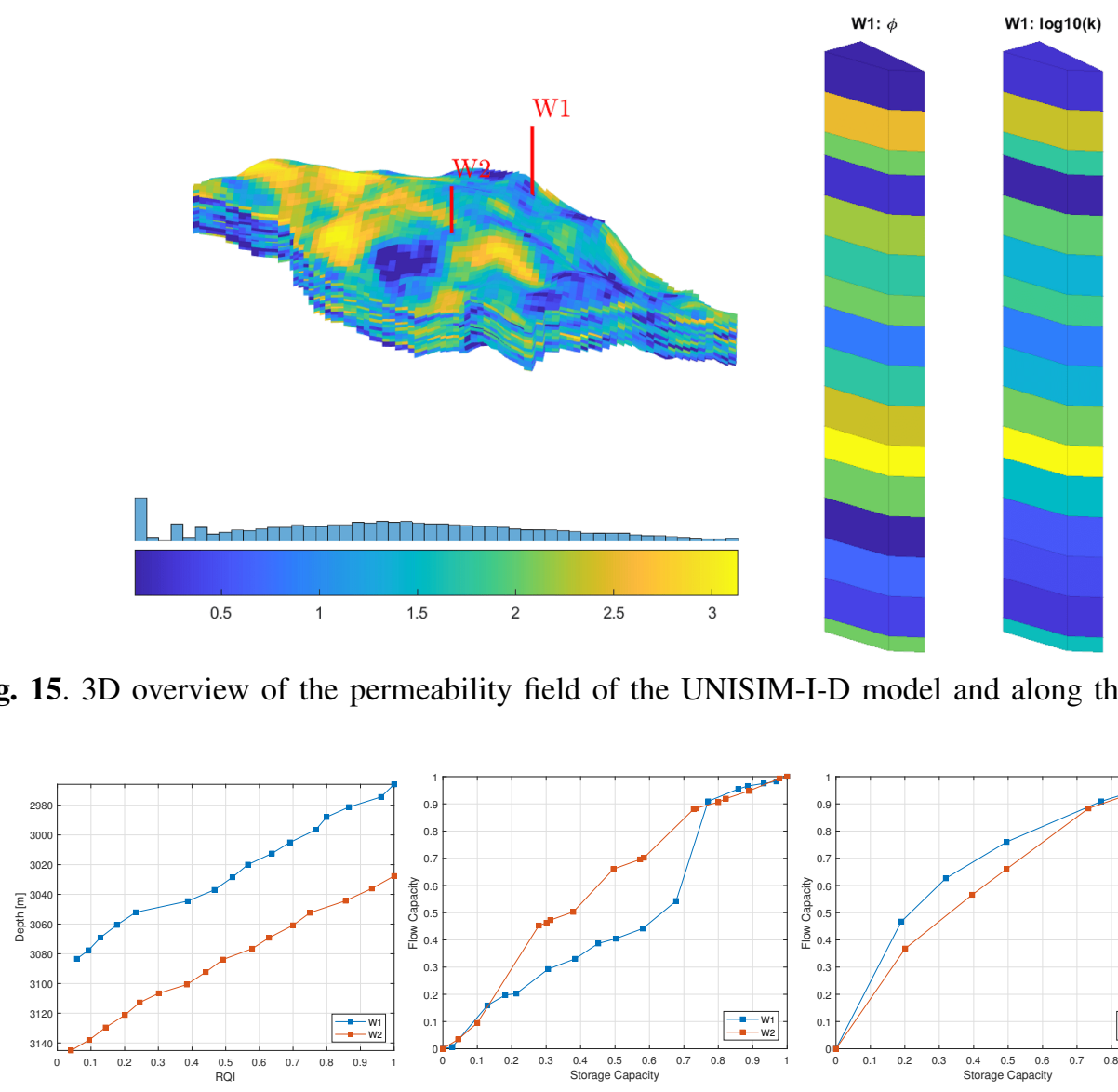

W2: $\phi$

W2: $\log 10(\mathrm{k})$
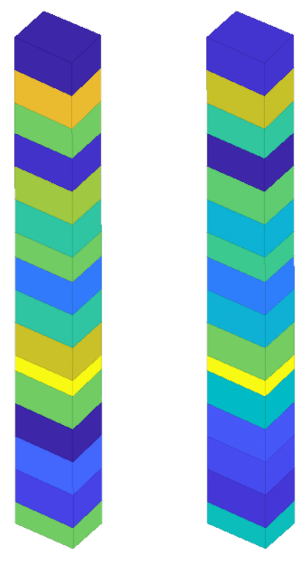

Fig. 15. 3D overview of the permeability field of the UNISIM-I-D model and along the perforated cells of two wells.

Fig. 16. NCRQIP, SMLP, MLP, and DSMLP for two arbitrary wells chosen over the UNISIM-I-D model (from left to right).

is well defined within the range $[0,5]$ of $F Z I$, thus indicating a flow unit. A slowly growing behavior appears in $W_{1}$ from $F Z I=4.0$ onward that seemingly aggregates a single unit. Empty bins in the histogram reflect numerical artifacts caused by undefined values at certain well layers.

We close the demonstration of GAWPS for this model by presenting the RPS-clustering map, FUS, and $\alpha(F U S)$ correspondences for the two sampled models in Fig. 18. Here, five clusters are defined per well. In particular, the highest average RPS in $W_{1}$ is around 2.4 near $3,041 \mathrm{~m}$, while it
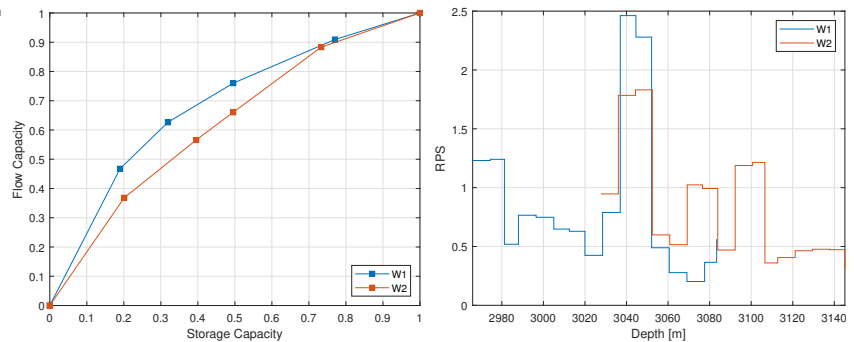

approaches 1.4 in $W_{2}$ close to $3,028 \mathrm{~m}$. Geometrical variations of the corner-point grid are seen in the FUS and $\alpha$ plots, where it is possible to see slight tilts and layers thinner than others. This shows that GAWPS is correctly handling the computation of arbitrary cell thicknesses.

\subsection{Dataset 3: UNISIM-II-D model}

UNISIM-II-D is a carbonate reservoir model focused on providing production strategy studies (considering deterministic and probabilistic approaches) that appeared after the UNISIM-I 


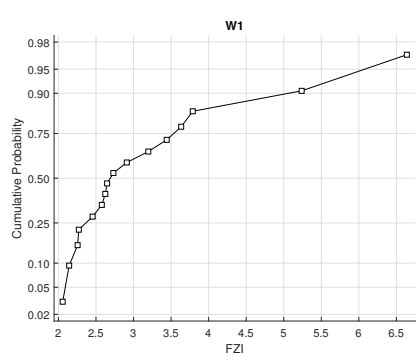

(a) $\operatorname{NPP}\left(W_{1}\right)$

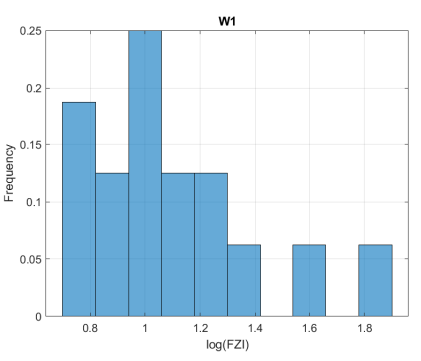

(b) Histogram $\left(W_{1}\right)$

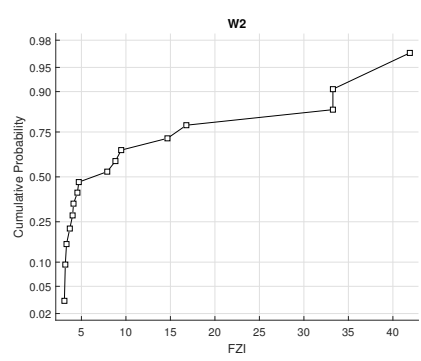

(c) $\operatorname{NPP}\left(W_{2}\right)$

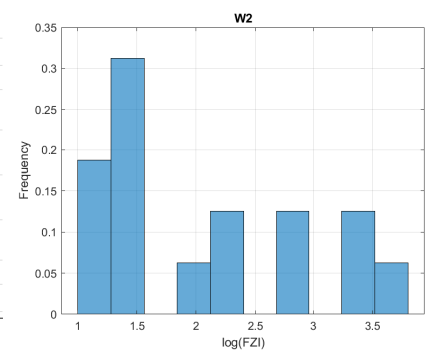

(d) Histogram $\left(W_{2}\right)$

Fig. 17. NPP and histogram plot applied to wells $W_{1}$ and $W_{2}$ for the UNISIM-I-D model.

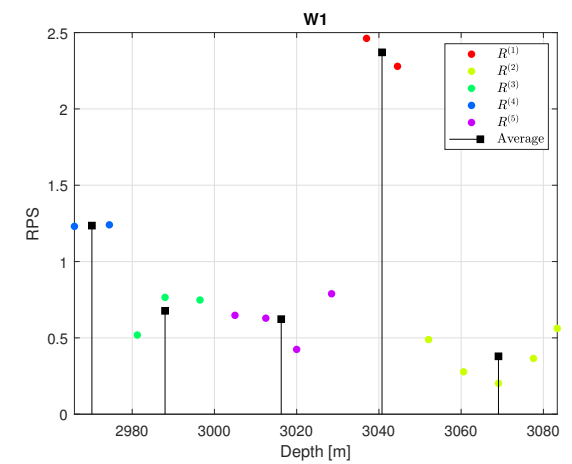

(a) $R P S$-clustering map $\left(W_{1}\right)$

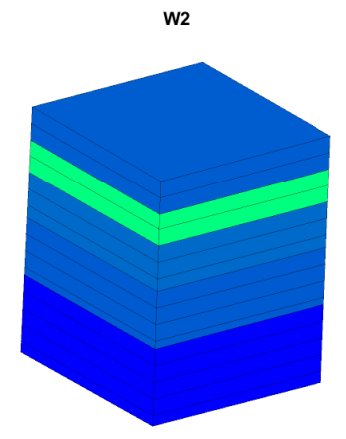

(d) $F U S\left(W_{2}\right)$

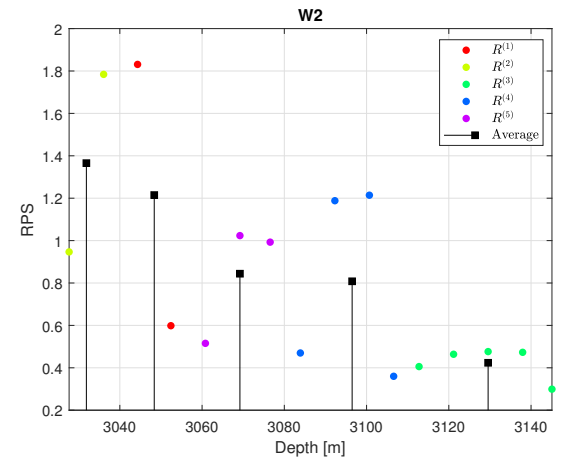

(b) RPS-clustering map $\left(W_{2}\right)$

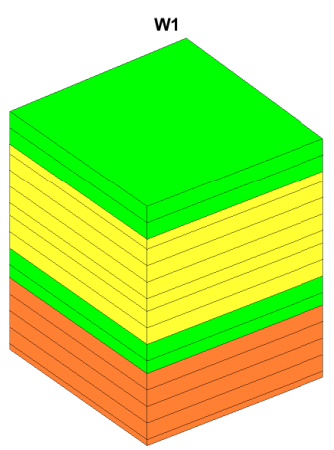

(e) $\alpha(F U S)\left(W_{1}\right)$

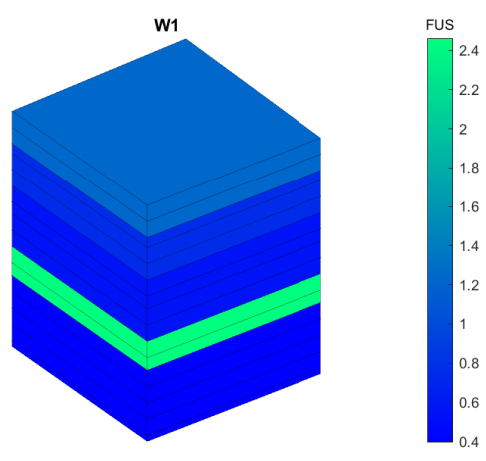

(c) $F U S\left(W_{1}\right)$

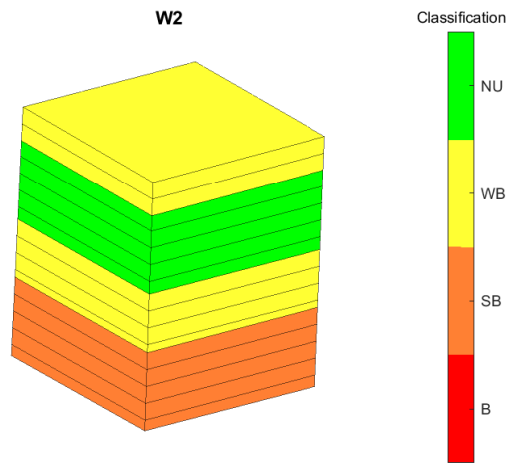

(f) $\alpha(F U S)\left(W_{2}\right)$

Fig. 18. RPS-clustering maps, FUS values, and speed zone classification $\alpha(F U S)$ for two wells over the UNISIM-I-D model.

family (Correia et al., 2015). In total there are 65000 active cells of size $100 \times 100 \times 8[\mathrm{~m}]$ for the corner-point grid of the model. The porosity field over the model is presented in the 3D view illustrated in Fig. 19.

CLP for this model is depicted in Fig. 20 and shows the degree of heterogeneity for both wells. UNSIM-II-D presents moderate heterogeneity at these locations. NCFCP is drawn in Fig. 21 and its purpose is similar to other methods that identify flow units through slopes. In particular, we can observe three units in $W_{2}$, the first one extending from 1,680 to $1,780 \mathrm{~m}$, the second one between $1,780 \mathrm{~m}$ and $1,800 \mathrm{~m}$, and the third one covering the depth range from 1,800 to $1,900 \mathrm{~m}$ approximately. On the other hand, a number of units superior to 3 would be found in $W_{2}$. Such diagnosis is coherent with CLP, since $W_{1}$ is more heterogeneous than $W_{2}$.

\subsection{Dataset 4: Norne model}

The Norne is a realistic black-oil model for an oilfield in the Norwegian Sea, which has a grid of faulty corner points with heterogeneous and anisotropic permeability. The model was developed to be a key benchmark for the petroleum industry.

The original benchmark is hosted and managed by NTNU/Norway, and the simulation model was later made available by the OPM initiative ${ }^{2}$. Fig. 22 shows the porosity distribution of the model.

Our analysis uses two wells that have very similar petrophysical characteristics; the Winland plot and NCFCP shown in Fig. 23 confirm this. In the former, the high concentration

\footnotetext{
${ }^{2}$ Available on: https://opm-project.org/?page_id=559.
} 

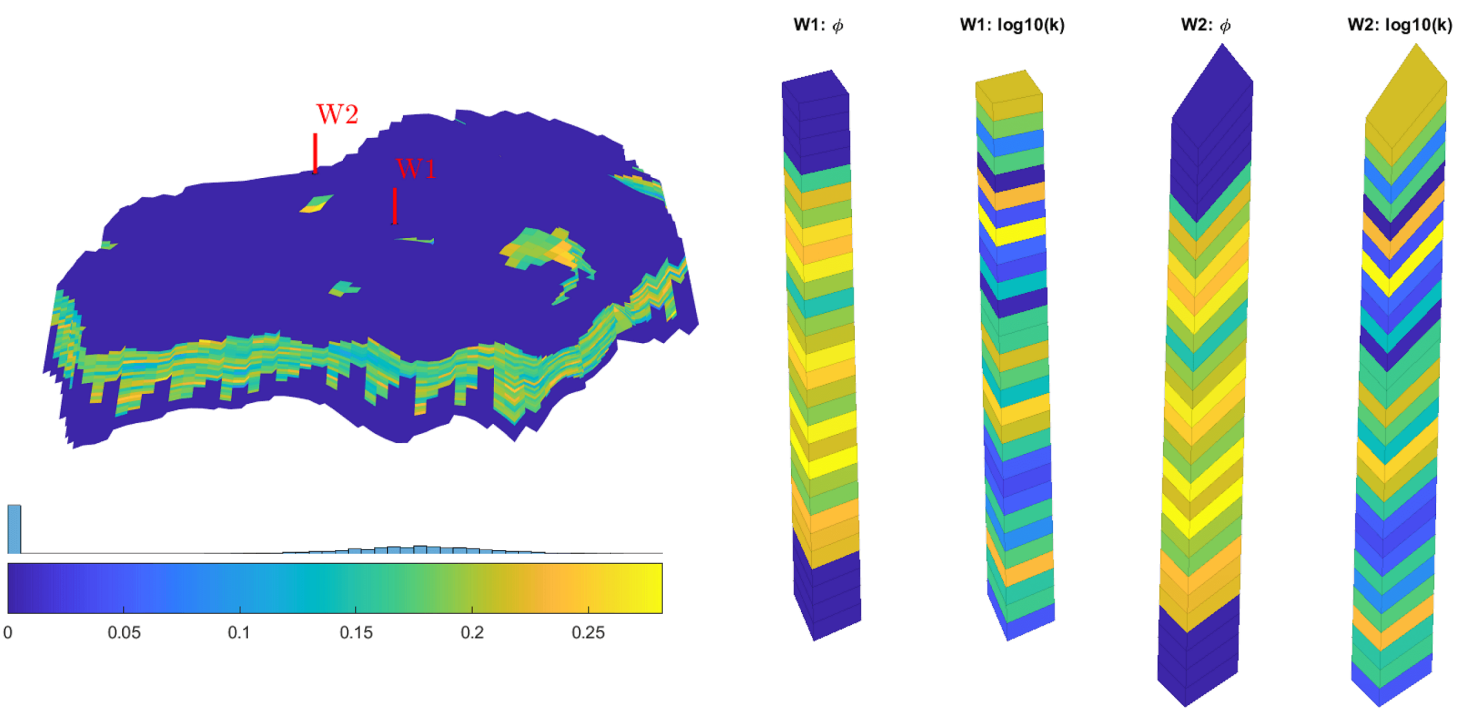

Fig. 19. 3D overview of the UNISIM-II-D model along with the porosity field and two wells.

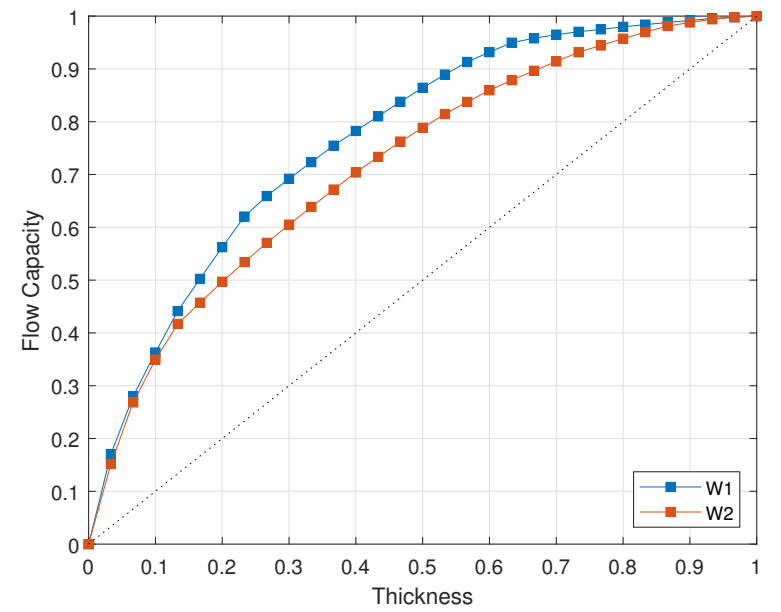

Fig. 20. Classic Lorenz plot for two random wells in UNISIMII-D.

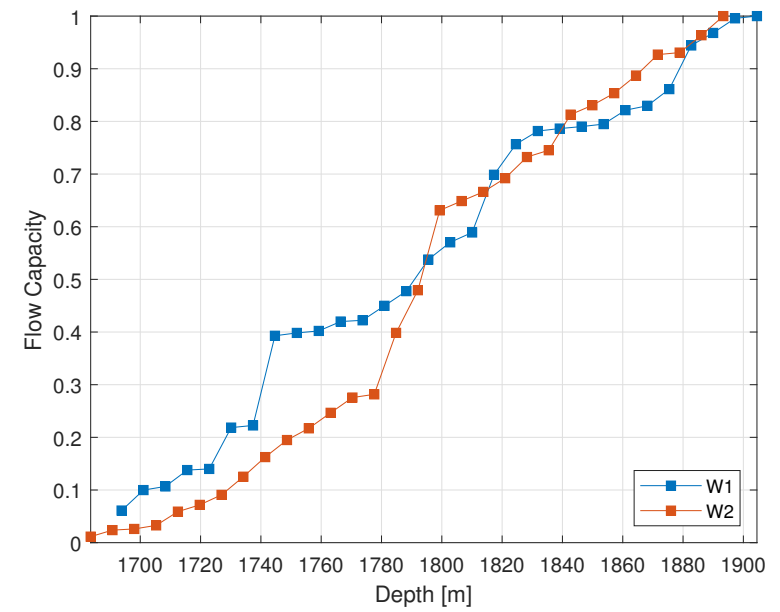

Fig. 21. Normalized cumulative flow capacity plot for two random wells over the UNISIM-II-D model.
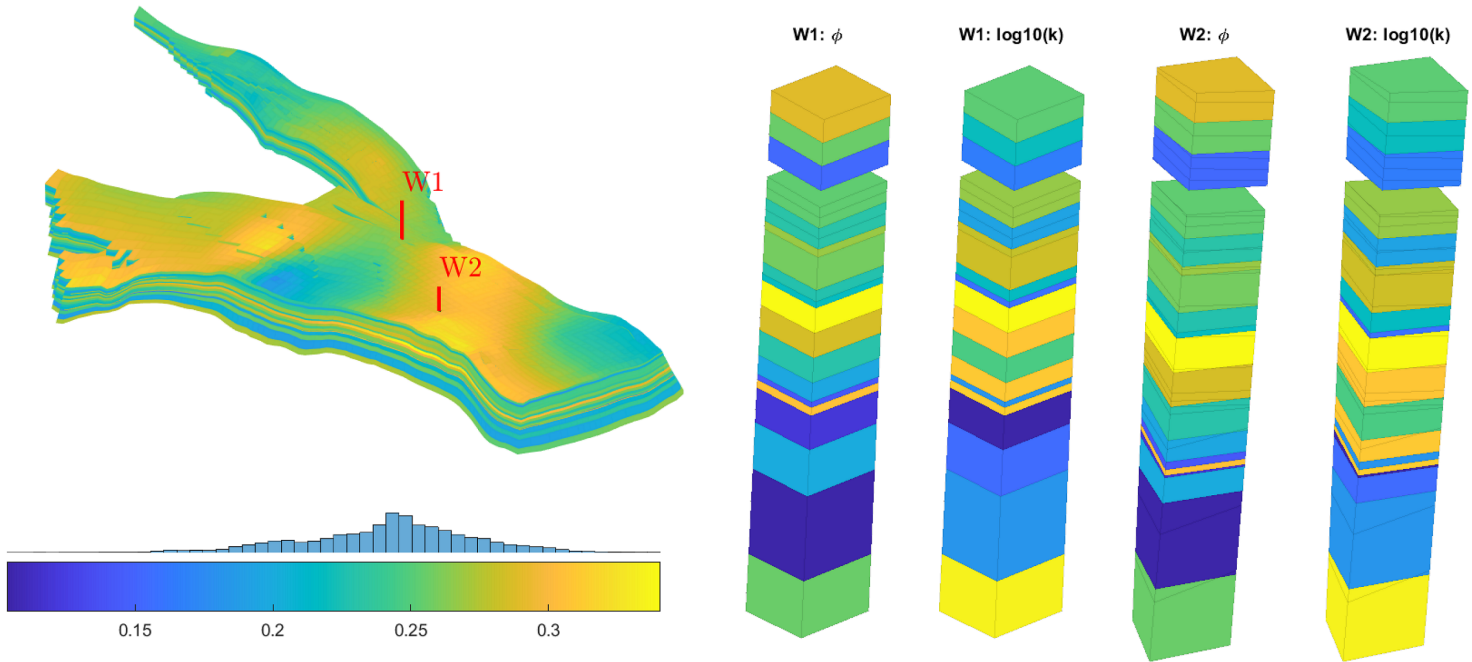

Fig. 22. Porosity field over the Norne model along with two sampled wells. 

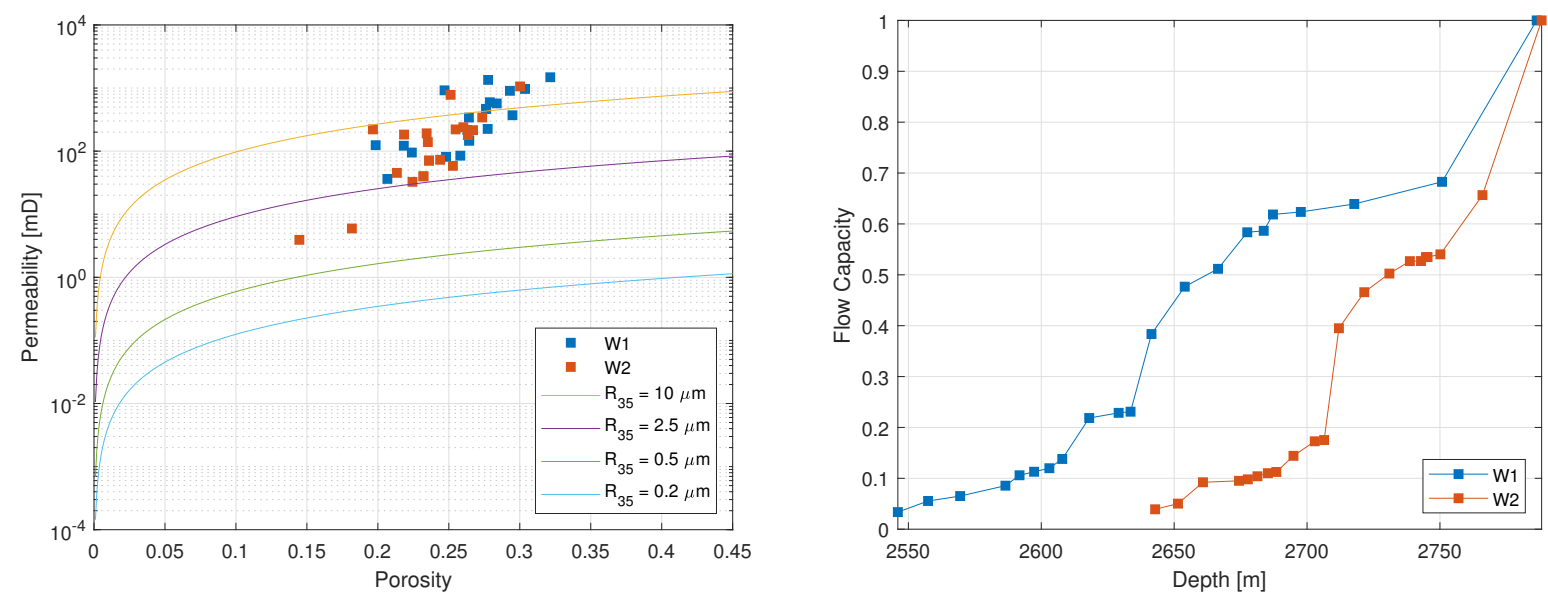

Fig. 23. Winland plot (left) and NCFCP (right) for two random wells over the Norne model.
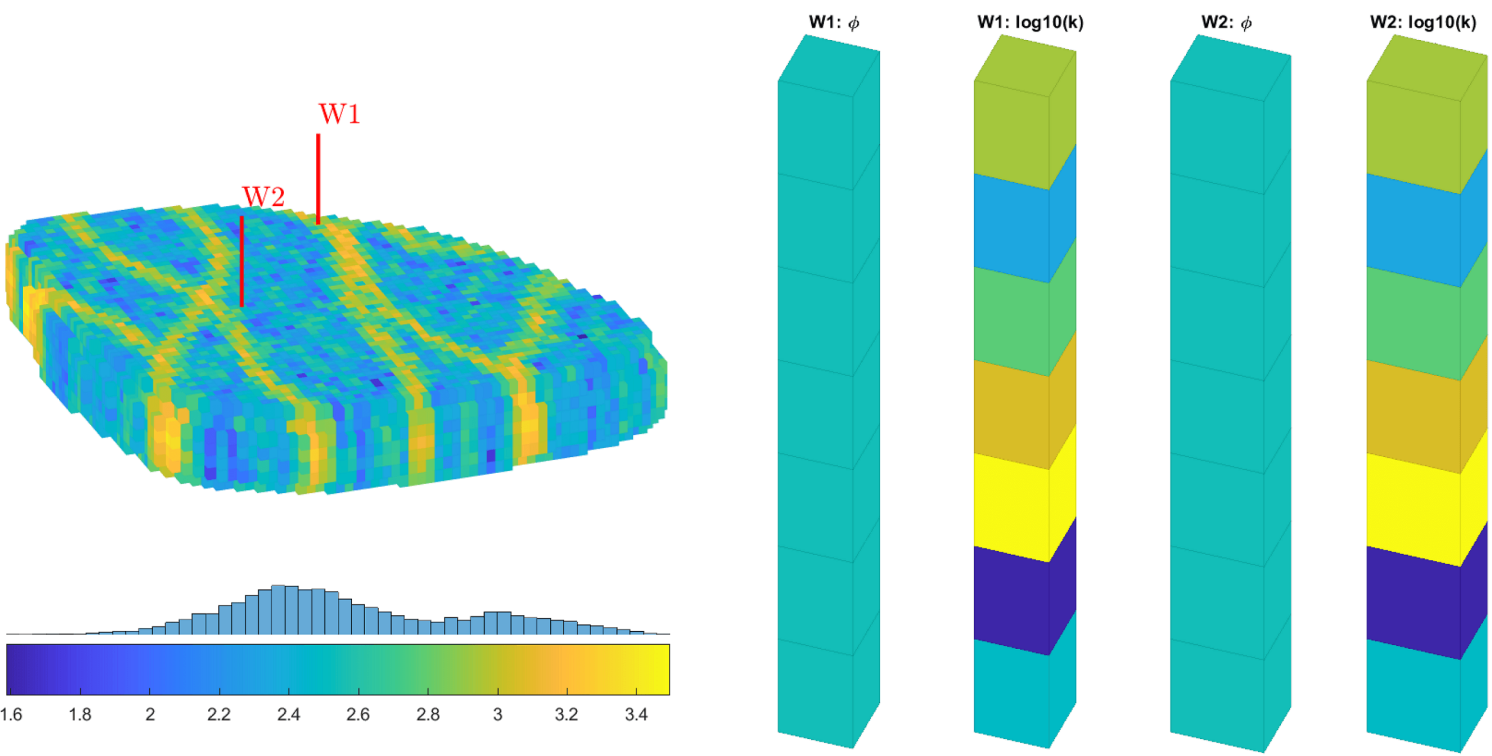

Fig. 24. Permeability field and sampled wells for the Egg model.

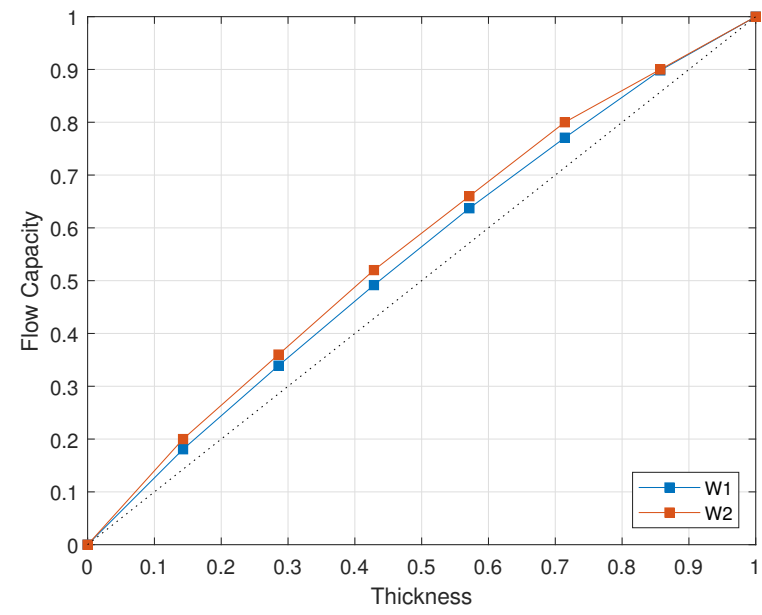

Fig. 25. Classic Lorenz plot for two random wells over the Egg model. Both straight lines close to the dashed line indicate that very low heterogeneity is observed depth-wise in the model. of points with porosity between 0.20 and 0.25 constitute megaporous ports. In the latter plot, we see well stratigraphy that reaches different depths. Naturally, it is arguable that both wells have crossed the same flow units.

\subsection{Dataset 5: Egg model}

The "Egg model" is a synthetic reservoir model consisting of an ensemble of 101 relatively small 3D realizations of a channelized oil reservoir produced under water-flooding conditions focused on flooding optimization and history matching.

It has $60 \times 60 \times 7$ grid cells, out of which the 18,553 active cells constitute an-egg-shaped volume (Jansen et al., 2014) as depicted in Fig. 24. As a result of the small size of this model, only CLP was deemed interesting, shown in Fig. 25. Given the construction of this model, we verify quasi-homogeneous behavior. 


\section{Conclusion}

We have introduced a package of computational routines useful for investigation of reservoir models. The module is structured to work jointly with an open-source software, whose user community increases day after day. The focus of the functions developed is to perform quantitative and qualitative graphical analysis of vertical well profiles.

We suggested the DSMLP as a helpful tool to study the local behavior of reservoir process speed and assist experts to identify flow unit boundaries and speed zones in petroleum reservoirs. Additionally, we adopted a classification function that identifies barriers, baffles, and speed zones after solving a clustering problem, so that petrophysical features can be directly displayed in terms of depth along the wellbore.

Through brief demonstrations, we explored the capabilities of the package in providing a neat visualization of the main features sought by geologists, petrophysicists, and reservoir modellers while surveying reservoirs from illustrative plots. In turn, the available code offers a compendium of analyses for flow unit delineation that can assist user interpretation. Although improvements are still necessary to reduce minor dependencies of built-in functions, most of the software capabilities can be seized.

\section{Nomenclature}

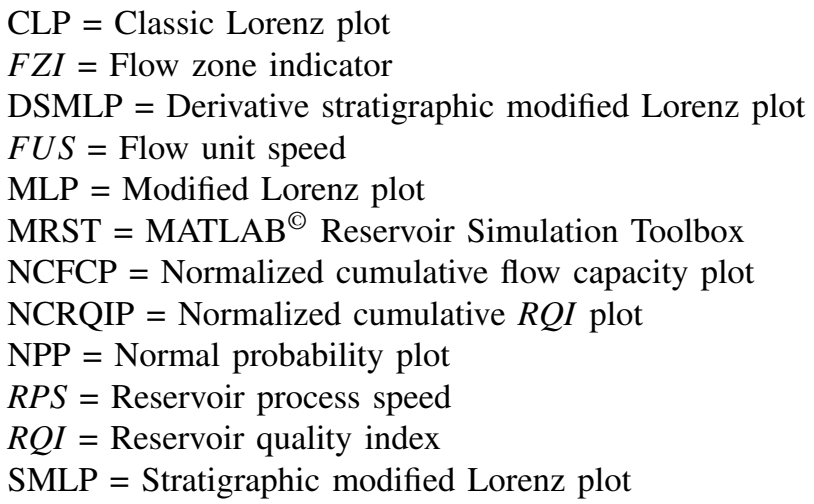

\section{Acknowledgement}

G. P. Oliveira thanks Dr. Francesca Watson (SINTEF) for reviewing this material. T. N. E. Rodrigues acknowledges the grant received from the Federal University of Paraíba under the Scientific Initiation Program.

\section{Code availability}

GAWPS can be downloaded directly from its project page on https://github.com/gcpeixoto/gawps.

\section{Conflict of interest}

The authors declare no competing interest.

Open Access This article is distributed under the terms and conditions of the Creative Commons Attribution (CC BY-NC-ND) license, which permits unrestricted use, distribution, and reproduction in any medium, provided the original work is properly cited.

\section{References}

Abbaszadeh, M., Fujii, H., Fujimoto, F., et al. Permeability prediction by hydraulic flow units-theory and applications. SPE Formation Evaluation, 1996, 11(4): 263-271.

Amaefule, J. O., Altunbay, M., Tiab, D., et al. Enhanced reservoir description: Using core and log data to identify hydraulic (flow) units and predict permeability in uncored intervals/wells. Paper SPE 26436 Presented at the SPE Annual Technical Conference and Exhibition, Houston, Texas, 3-6 October, 1993.

Avansi, G. D., Schiozer, D. J. UNISIM-I: Synthetic model for reservoir development and management applications. International Journal of Modeling and Simulation for the Petroleum Industry, 2015, 9(1): 21-30.

Benham, P., Freeman, M., Zhang, I., et al. The function of baffles within heavy oil reservoir and their impact on field development: A case study from kuwait. Paper SPE 193775 Presented at the SPE International Heavy Oil Conference and Exhibition, Kuwait City, Kuwait, 10-12 December, 2018.

Cannon, S. Petrophysics: A Practical Guide. New York, USA, John Wiley \& Sons, 2015.

Christie, M. A., Blunt, M. J. Tenth SPE comparative solution project: A comparison of upscaling techniques. Paper SPE 66599 Presented at the SPE Reservoir Simulation Symposium, Houston, Texas, 11-14 February, 2001.

Correia, M., Hohendorff, J., Gaspar, A. T., et al. UNISIM-II-D: Benchmark case proposal based on a carbonate reservoir. Paper SPE 177140 Presented at the SPE Latin American and Caribbean Petroleum Engineering Conference, Quito, Ecuador, 18-20 November, 2015.

Craig, F. F. The Reservoir Engineering Aspects of Waterflooding, Volume 3. New York, USA, Society of Petroleum Engineers of AIME, 1971.

Cunningham, K. J., Sukop, M. C., Huang, H., et al. Prominence of ichnologically influenced macroporosity in the karst biscayne aquifer: Stratiform "super-k" zones. Geological Society of America Bulletin, 2009, 121(1-2): 164-180.

Dake, L. P. The Practice of Reservoir Engineering (Revised Edition). Amsterdam, Netherlands, Elsevier, 2001.

Ebanks Jr., W. J., Scheihing, M. H., Atkinson, C. D. Flow units for reservoir characterization: Part 6. Geological methods, in ME 10: Development Geology Reference Manual, edited by D. Morton-Thompson and A. M. Woods, American Association of Petroleum Geologists, Tulsa, pp. 282-285, 1992.

Fanchi, J. Integrated Reservoir Asset Management: Principles and Best Practices. Houston, USA, Gulf Professional Publishing, 2010.

Gunter, G., Sahar, M. Y., Viro, E., et al. Introducing a ten-step integrated petrophysical rock type verification process that combines deterministic methods, saturation height modeling, advanced flow units and IPSOM. Paper SPE 193147 Presented at the Abu Dhabi International Petroleum Exhibition \& Conference, Abu Dhabi, UAE, 12-15 November, 2018.

Gunter, G. W., Allen, D. F., Marché, O. A., et al. Challenges 
of determining and comparing reservoir storage and flow properties using deterministic petrophysical rock types via multiple pore throat radius indicators. Paper SPWLA 2017-OO Presented at the SPWLA 58th Annual Logging Symposium, Oklahoma City, Oklahoma, USA, 17-21 June, 2017.

Gunter, G. W., Finneran, J. M., Hartmann, D. J., et al. Early determination of reservoir flow units using an integrated petrophysical method. Paper SPE 38679 Presented at the SPE Annual Technical Conference and Exhibition, San Antonio, Texas, 5-8 October, 1997a.

Gunter, G. W., Pinch, J. J., Finneran, J. M., et al. Overview of an integrated process model to develop petrophysical based reservoir descriptions. Paper SPE 38748 Presented at the SPE Annual Technical Conference and Exhibition, San Antonio, Texas, 5-8 October, 1997b.

Gunter, G. W., Viro, E. J., Wolgemuth, K. Identifying value added opportunities by integrating well log interpretation, petrophysical rock types and flow units; introducing a new multi-component stratigraphic modified lorenz method. Paper SPWLA 2012-170 Presented at the SPWLA 53rd Annual Logging Symposium, Cartagena, Colombia, 16-20 June, 2012.

Hatampour, A., Schaffie, M., Jafari, S. Hydraulic flow units, depositional facies and pore type of Kangan and Dalan Formations, South Pars Gas Field, Iran. Journal of Natural Gas Science and Engineering, 2015, 23: 171-183.

Hearn, C. L., Ebanks Jr., W. J., Tye, R. S., et al. Geological factors influencing reservoir performance of the Hartzog Draw field, Wyoming. Journal of Petroleum Technology, 1984, 36(8): 1335-1344.

Jansen, J.-D., Fonseca, R.-M., Kahrobaei, S., et al. The egg model-a geological ensemble for reservoir simulation. Geoscience Data Journal, 2014, 1(2): 192-195.

Kolodzie Jr., S. Analysis of pore throat size and use of the waxman-smits equation to determine ooip in Spindle field, Colorado. Paper SPE 9382 Presented at the SPE Annual Technical Conference and Exhibition, Dallas, Texas, 21-24 September, 1980.

Lie, K.-A. An Introduction to Reservoir Simulation Using MATLAB/GNU Octave: User Guide for the MATLAB Reservoir Simulation Toolbox (MRST). Cambridge, UK, Cambridge University Press, 2019.

Mahjour, S. K., Al-Askari, M. K. G., Masihi, M. Identification of flow units using methods of testerman statistical zonation, flow zone index, and cluster analysis in Tabnaak gas field. Journal of Petroleum Exploration and Production Technology, 2016, 6(4): 577-592.

Newsham, K. E., Rushing, J. A. An integrated work-flow model to characterize unconventional gas resources: Part Igeological assessment and petrophysical evaluation. Paper SPE 71351 Presented at the SPE Annual Technical Conference and Exhibition, New Orleans, Louisiana, 30 September-3 October, 2001.

Oliveira, G. P., Roque, W. L., Araújo, E. A., et al. Competitive placement of oil perforation zones in hydraulic flow units from centrality measures. Journal of Petroleum Science and Engineering, 2016, 147: 282-291.

Oliveira, G. P., Santos, M. D., Roque, W. L. Constrained clustering approaches to identify hydraulic flow units in petroleum reservoirs. Journal of Petroleum Science and Engineering, 2020, 186: 106732.

Pittman, E. D. Relationship of porosity and permeability to various parameters derived from mercury injectioncapillary pressure curves for sandstone. AAPG Bulletin, 1992, 76(2): 191-198.

Rahimpour-Bonab, H., Enayati-Bidgoli, A., Navidtalab, A., et al. Appraisal of intra reservoir barriers in the PermoTriassic successions of the Central Persian Gulf, Offshore Iran. Geologica Acta, 2014, 12(1): 87-107.

Rushing, J. A., Newsham, K. E. An integrated work-flow model to characterize unconventional gas resources: Part II-formation evaluation and reservoir modeling. Paper SPE 71352 Presented at the SPE Annual Technical Conference and Exhibition, New Orleans, Louisiana, 30 September-3 October, 2001.

Siddiqui, S., Okasha, T. M., Funk, J. J., et al. New representative sample selection criteria for special core analysis. Paper Presentation at the International Symposium of the Society of Core Analysts held in Pau, France, 21-24 September, 2003.

Svirsky, D., Ryazanov, A., Pankov, M., et al. Hydraulic flow units resolve reservoir description challenges in a Siberian oil field. Paper SPE 87056 Presented at the SPE Asia Pacific Conference on Integrated Modelling for Asset Management, Kuala Lumpur, Malaysia, 29-30 March, 2004.

Zhou, W., Liang, S., Ma, D., et al. Statistical verification of hydraulic units in a heterogeneous reservoir of the Liaohe Oilfield. Journal of Earth Science, 2014, 25(6): 991-1002. 